\title{
A MODEL IN WHICH GCH HOLDS AT SUCCESSORS BUT FAILS AT LIMITS
}

\author{
JAMES CUMMINGS
}

\begin{abstract}
Starting with GCH and a $\mathscr{P}_{3} \kappa$-hypermeasurable cardinal, a model is produced in which $2^{\lambda}=\lambda^{+}$if $\lambda$ is a successor cardinal and $2^{\lambda}=\lambda^{++}$if $\lambda$ is a limit cardinal. The proof uses a Reverse Easton extension followed by a modified Radin forcing.
\end{abstract}

\section{INTRODUCTION}

1.1. Historical remarks and statement of the main result. The continuum problem is an old one, dating back to Cantor and his statement of the Continuum Hypothesis in [Ca]. Put in a modern form which might have puzzled Cantor, the problem is to determine which behaviours of the continuum function $\kappa \mapsto 2^{\kappa}$ are consistent with ZFC. Throughout this paper ZFC will be the base set theory, though as we see below strong set-theoretic hypotheses will play an essential role in the result.

Before Gödel, progress on the continuum problem was made by the descriptive set theorists, who showed that certain easily definable sets of reals could not be counterexamples to $\mathrm{CH}$. Gödel [G] took the major step forward of showing that in a certain submodel of $V$, the constructible universe $L$, the following Generalised Continuum Hypothesis is true (here CARD denotes the class of cardinals):

$(\mathrm{GCH})$

$$
\forall \kappa \in \mathrm{CARD} \quad 2^{\kappa}=\kappa^{+} \text {. }
$$

Then in 1963 Cohen [Co] initiated the method of forcing and showed that the negation of $\mathrm{CH}$ was also consistent with $\mathrm{ZFC}$, making $\mathrm{CH}$ the first statement of "natural" mathematics to be shown undecidable in set theory. This breakthrough was quickly followed by the work of Easton [E], who showed that if GCH holds in $V$ and $F:$ REG $\rightarrow$ CARD (where REG is the class of regular cardinals) is a class function such that

$$
\begin{aligned}
& \forall \kappa \in \mathrm{REG} \quad F(\kappa)>\kappa, \\
& \forall \kappa, \lambda \in \mathrm{REG} \quad \kappa<\lambda \Rightarrow F(\kappa) \leq F(\lambda), \\
& \forall \kappa \in \mathrm{REG} \operatorname{cf}(F(\kappa))>\kappa,
\end{aligned}
$$

Received by the editors June 2, 1989 and, in revised form, March 7, 1990.

1980 Mathematics Subject Classification (1985 Revision). Primary 03E35; Secondary 03E55.

Key words and phrases. Radin forcing, singular cardinals problem, large cardinals.

The author would like to thank Adrian Mathias and Hugh Woodin, who supervised the doctoral thesis from which this result is taken, and the Science and Engineering Research Council of Great Britain for their support. 
then there exists a class forcing preserving all cardinals and cofinalities such that in the extension

$$
\forall \kappa \in \mathrm{REG} \quad 2^{\kappa}=F(\kappa) .
$$

That is, the values of the continuum function at regular cardinals are highly independent of each other, and can be "anything reasonable."

It is worth noticing that all the consistency results mentioned so far are relative to $\mathrm{ZFC}$, that is, are results of the form "Con(ZFC) implies Con $(Z F C+\phi)$," where $\phi$ is some statement of set theory.

The first connection between large cardinals and the continuum function was made slightly prior to the invention of forcing by Scott [Sc], who used his technique of taking ultrapowers of $V$ to show that if $\kappa$ is measurable, $U$ is a normal measure on $\kappa$, and $\left\{\alpha<\kappa \mid 2^{\alpha}=\alpha^{+}\right\} \in U$ then $2^{\kappa}=\kappa^{+}$. So sufficiently strong large cardinals can impose some structure on the behaviour of the continuum function.

For some time after Easton's work it was felt that Easton's result should generalise to any "reasonable" function $F:$ CARD $\rightarrow$ CARD, and that all that was needed were more sophisticated techniques for forcing over models of ZFC; but then Silver [Si1] proved some theorems showing that if $\kappa$ is a singular strong limit cardinal of uncountable cofinality then there is a strong connection between the behaviour of the continuum function below $\kappa$ and the value of $2^{\kappa}$. For example if $\mathrm{GCH}$ fails at $\kappa$ then it already fails on a closed unbounded set of cardinals less than $\kappa$ (the parallel with Scott's result is not coincidence; Silver's proof goes via a generic ultrapower by an ultrafilter extending the club filter).

More surprises were in store; let $\mathrm{SCH}$ (Singular Cardinals Hypothesis) be the assertion that if $\kappa$ is singular then $2^{\kappa}$ is the least cardinal $\lambda \geq 2^{<\kappa}$ with $\operatorname{cf}(\lambda)>\kappa$. This would imply that the continuum function was determined by its behaviour on the class of regular cardinals. Jensen [DJ] was inspired by seeing Silver's result to show that if $0^{\sharp}$ does not exist then $\mathrm{SCH}$ is true: however by work of Silver and Prikry (see [Si2, P]) it was known that if the existence of a supercompact cardinal is consistent then so is the failure of SCH (use Reverse Easton forcing to get a measurable $\kappa$ such that $2^{\kappa}=\kappa^{++}$, then use Prikry forcing to make $\kappa$ have cofinality $\omega ; \kappa$ becomes a rather large singular strong limit cardinal at which $\mathrm{GCH}$ fails). So large cardinals are essential for an understanding of the continuum function.

It is natural to ask about the situation at small singular cardinals. Magidor showed in [Ma1, Ma2] that (among other things)

(1) $\operatorname{Con}\left(\mathrm{ZFC}+\mathrm{GCH}+\right.$ there is $\kappa \kappa^{++}$-supercompact) implies $\mathrm{Con}\left(\aleph_{\omega}\right.$ is strong limit and $\left.2^{\aleph_{\omega}}=\aleph_{\omega+2}\right)$, and

(2) $\operatorname{Con}(\mathrm{ZFC}+$ there exist $\kappa<\lambda$ with $\kappa$ supercompact and $\lambda$ huge) implies Con (GCH holds below $\aleph_{\omega}$ and $\left.2^{\aleph_{\omega}}=\aleph_{\omega+2}\right)$.

From (1) SCH can fail at the smallest singular cardinal of them all; from (2) no direct analogue of the theorem from [Si1] mentioned above can hold.

There is a large gap in strength between $0^{\sharp}$ and a supercompact cardinal. At one end Dodd and Jensen [D] invented the core model and showed that failure of SCH has consistency strength greater than that of a measurable cardinal, and following this line of research Mitchell [Mi2] obtained the consistency of measurable cardinals of high order from the failure of $\mathrm{SCH}$. At the other end 
Woodin [W] showed how to get Magidor's result (1) from the consistency of a $\mathscr{P}_{2} \kappa$-hypermeasurable cardinal. Shelah obtained some bounds [Sh1, Chapter XIII] for singular cardinals of cofinality $\omega$ : for example if $\aleph_{\omega}$ is strong limit then $2^{\aleph_{\omega}}<\aleph_{\left(2^{\left.\aleph_{0}\right)^{+}}\right.}$. Shelah also showed [Sh2] that $\aleph_{\omega}$ being strong limit was consistent with $2^{\aleph_{\omega}}$ being any successor cardinal below $\aleph_{\omega_{1}}$, using a supercompact, and Magidor extended this in unpublished work to get a similar result with $\mathrm{GCH}$ holding below $\aleph_{\omega}$.

Another line of development has involved global results. Foreman and Woodin [FW] showed that starting from slightly more than a supercompact it is consistent that the GCH fail everywhere, and Woodin then produced a model where $\forall \kappa \quad 2^{\kappa}=\kappa^{++}$. Woodin later showed that hypermeasurability-type hypotheses suffice for these results.

This paper, which is basically a revised version of Chapter 2 of my doctoral thesis [C], also proves a global result: if a $\mathscr{P}_{3} \kappa$-hypermeasurable cardinal is consistent then so is

\section{$\mathrm{ZFC}+\mathrm{GCH}$ holds at all successors and fails at all limits.}

The final model is of the form $V_{\kappa}^{*}$, where $\kappa$ is a $\mathscr{P}_{2} \kappa$-hypermeasurable in $V^{*}$, so the model contains many smallish large cardinals.

The proof combines Radin forcing with ideas from unpublished work of Woodin, in which he shows that Magidor's result (2) mentioned above can also be done from a $\mathscr{P}_{2} \kappa$-hypermeasurable.

Added in proof. Magidor and Gitik [GM] have recently determined the exact strength of the failure of SCH. A sample result is that failure at $\aleph_{\omega}$ is equiconsistent with $o(\kappa)=\kappa^{++}$. Their methods will also do Magidor's result (2) from this hypothesis (and indeed the stronger result with GCH up to $\aleph_{\omega}$ and a countable jump at $\aleph_{\omega}$ from less than a strong cardinal) but are very specific to cardinals of cofinality $\omega$ and do not seem to mix with Radin forcing in any straightforward way.

Also Shelah has proved the surprising theorem that if $2^{\aleph_{0}}<\aleph_{\omega}$ then $\aleph_{\omega}^{\aleph_{0}}<$ $\aleph_{\omega_{4}}$, and shown that some new idea is needed to make a model in which $\aleph_{\omega}$ is strong limit and $2^{\aleph_{\omega}}>\aleph_{\omega_{1}}$.

1.2. Background material. In this subsection we establish our terminology and notation; we also give proofs for some facts which we need in the Reverse Easton construction of $\S 2$. These facts are part of the folk literature of set theory, but we give the proofs here to make the exposition self-contained.

The set-theoretic notation used is mostly that of $[\mathrm{J}]$, with the caveat that the forcing terminology is that of Kunen's reference [K]; in particular, " $\kappa$-closed" means "having lower bounds for decreasing sequences of length less than $\kappa$." " $\kappa$-dense" means that the intersection of fewer than $\kappa$ dense open sets is dense. (set theoretic term $)_{M}$ means the result of computing the term in the model $M$, and when we write for example

$$
M \vDash \operatorname{cf}(\lambda)=\kappa^{++}
$$

we mean that $\operatorname{cf}_{M}(\lambda)=\kappa_{M}^{++}$. If $P$ is a poset and $p \in P$ then $P\lceil p$ is used to denote $\left\{q \in P \mid q \leq_{P} p\right\}$ with the inherited partial ordering. When we say that two posets are isomorphic we mean there is an order preserving map from 
one to the other with dense domain and range (this is enough to make them equivalent from the point of view of forcing).

Certain forcing notions will be used very heavily; if $\kappa$ is regular, $\lambda \geq \kappa$ is a cardinal, and $\mu>\kappa$ is inaccessible then

(1) $\operatorname{Add}(\kappa, \lambda)$ is the poset of partial functions from $\kappa \times \lambda$ to 2 with supports of size less than $\kappa$. We regard it as adding $\lambda$ generic subsets of $\kappa$. It is $\kappa$-closed and $\left(2^{<\kappa}\right)^{+}$-c.c., and will make $2^{<\kappa}$ have cardinality $\kappa$ without adding bounded subsets to $\kappa$.

(2) $\operatorname{Coll}(\kappa, \lambda)$ is the poset of partial functions from $\kappa$ to $\lambda$ with supports of size less than $\kappa$. It will collapse $\lambda$ to be of cardinality $\kappa$.

(3) $\operatorname{Coll}(\kappa,<\mu)$ is the poset of partial functions $f: \kappa \times \mu \leadsto \mu$, where $f$ has support of size less than $\kappa$ and $\forall \alpha \forall \beta f(\alpha, \beta)<\beta$. It is $\kappa$-closed and $\mu$-c.c., and makes $\mu$ into the successor of $\kappa$.

Many well-known facts about iterated forcing will be used without comment; we refer the reader to Baumgartner's article [B].

1. Extenders. An extender is a device for describing an elementary embedding between models of set theory; there is a good account in [MS] but our conventions differ rather from those of Martin and Steel. The reader of [MS] should have no trouble in providing proofs for the assertions we make here.

Definition. Let $a, b$ be finite sets of ordinals with $a \subseteq b$. Suppose

$$
b=\left\{\alpha_{1}, \ldots, \alpha_{n}\right\}_{<}, \quad a=\left\{\alpha_{l_{1}}, \ldots, \alpha_{l_{m}}\right\}_{<\cdot}
$$

Define $f_{a b}:[\mathrm{ON}]^{n} \longrightarrow[\mathrm{ON}]^{m}$ by

$$
f_{a b}:\left\{\lambda_{1}, \ldots, \lambda_{n}\right\}_{<} \mapsto\left\{\lambda_{l_{1}}, \ldots, \lambda_{l_{m}}\right\}_{<\cdot}
$$

Definition. Let $M$ be a transitive model of ZFC, let $\kappa$ be a cardinal of $M$, and let $\lambda>\kappa$ be an ordinal. Then $E$ is a $(\kappa, \lambda)$-M-extender iff

$$
E=\left\langle E_{a}: a \in[\lambda]^{<\omega}\right\rangle,
$$

where for some ordinal $\mu$

(1) For all $a \in[\lambda]^{<\omega} \quad E_{a}$ is a $M$ - $\kappa$-complete $M$-ultrafilter on $[\mu]^{|a|}$.

(2) For all $a, b \in[\lambda]^{<\omega}$, if $a \subset b$ and $f_{a b}:[\mu]^{|b|} \rightarrow[\mu]^{|a|}$ is defined as above, then

$$
\forall A A \in E_{a} \Leftrightarrow\left\{x \mid f_{a b}(x) \in A\right\} \in E_{b} .
$$

(3) The limit ultrapower of $M$ by the system $E$ (which exists by (1) and (2) and standard facts about limit ultrapowers) is well founded.

(4) If $j_{E}: M \longrightarrow N$ is the limit ultrapower embedding then

(a) $\operatorname{crit}\left(j_{E}\right)=\kappa$.

(b) $\lambda \leq j_{E}(\mu)$.

(c) For all $a \in[\lambda]^{<\omega} \quad X \in E_{a} \Leftrightarrow a \in j_{E}(X)$.

(d) $N=\left\{j_{E}(f)(a) \mid a \in[\lambda]^{<\omega} \wedge f \in M \wedge f:[\mu]^{|a|} \rightarrow M\right\}$.

Definition. If $E$ is an extender as above then the critical point of $E$ is $\kappa$, the length of $E$ is $\lambda$, and the width of $E$ is $\mu$.

The motivation for this definition is that if we have transitive models of ZFC $M, N$, and an elementary embedding $i: M \rightarrow N$ such that $\lambda \leq i(\mu)$, then we can define a $(\operatorname{crit}(i), \lambda)-M$-extender of width $\mu$ by

$$
X \in E_{a} \Leftrightarrow a \in i(X)
$$


for $a \in[\lambda]^{<\omega}$. The embedding $i$ will factor as $k \circ j_{E}$, where $\operatorname{crit}(k) \geq \lambda$ and $k:\langle f, a\rangle_{E} \mapsto i(f)(a) . \quad i_{E}$ is an approximation to $i$ which improves as $\lambda$ increases.

We list some facts.

Fact 1. If $G C H$ holds and $j: V \rightarrow M$ is an elementary embedding into a transitive inner model $M$, with critical point $\kappa$, such that ${ }^{\kappa} M \subset M$ and $V_{\kappa+n} \subset M$ (a so-called $\mathscr{P}_{n} \kappa$-hypermeasurable embedding) then the extender of length $\kappa^{+n}$ approximating $j$ has width $\kappa$ and gives rise to a $\mathscr{P}_{n} \kappa$-hypermeasurable embedding.

Fact 2. Let $G C H$ hold, and let $j: V \rightarrow M$ be a $\mathscr{P}_{n} \kappa$-hypermeasurable embedding generated by $a\left(\kappa, \kappa^{+n}\right)$-V-extender. Factor $j$ as $k \circ j_{0}$, where $j_{0}: V \rightarrow M_{0}$ is the ultrapower of $V$ by $\{X \mid \kappa \in j(X)\}$. Then $k$ is generated by $a\left(\kappa_{M_{0}}^{++}, \kappa^{+n}\right)$ $M_{0}$-extender of width $\kappa_{M_{0}}^{+n}$.

Fact 3. If $E \in V$ is a $V$-extender then for every ordinal $\alpha$ the $\alpha$ th iterate of $V$ by $E$ exists and is well founded.

We make the remark that GCH was critical to the truth of Facts 1 and 2; later on we will construct a model in which $\kappa$ is $\mathscr{P}_{2} \kappa$-hypermeasurable and $2^{\kappa}=\kappa^{++}$, and there we will need a $\left(\kappa, \kappa^{+3}\right)$-extender to describe the associated elementary embedding. This is natural enough, since in this instance we have $\left|V_{\kappa+2}\right|=\kappa^{+3}$.

2. Extending embeddings. We collect some information about forcing and elementary embeddings, for use in the Reverse Easton constructions of $\S 2$.

Fact 1. Let $M, N$ be inner models of ZFC, $j: M \rightarrow N$ an elementary embedding, and $P \in M$ a notion of forcing. Let $G$ be $P$-generic over $M, H$ $j(P)$-generic over $N$, and suppose

$$
\forall p \in P \quad p \in G \Rightarrow j(p) \in H .
$$

Then $j$ can be extended to $j^{*}: M[G] \rightarrow N[H]$, where

$$
j^{*}: i_{G}(\dot{\tau}) \mapsto i_{H}(j(\dot{\tau}))
$$

and $j^{*}$ is still elementary.

Proof. Let $\phi\left(x_{1}, \ldots, x_{n}\right)$ be a formula of set theory with free variables among $x_{1}, \ldots, x_{n}$.

$$
\begin{aligned}
M[G] & \vDash \phi\left(i_{G}\left(\dot{\tau}_{1}\right), \ldots, i_{G}\left(\dot{\tau}_{n}\right)\right) \\
& \Rightarrow \exists p \in G \quad p \Vdash_{P}^{M} \phi\left(\dot{\tau}_{1}, \ldots, \dot{\tau}_{n}\right) \\
& \Rightarrow \exists q \in H \quad q \Vdash_{j(P)}^{N} \phi\left(j\left(\dot{\tau}_{1}\right), \ldots, j\left(\dot{\tau}_{n}\right)\right) \\
& \Rightarrow N[H] \vDash \phi\left(i_{H}\left(j\left(\dot{\tau}_{1}\right)\right), \ldots, i_{H}\left(j\left(\dot{\tau}_{n}\right)\right)\right) .
\end{aligned}
$$

Letting $\phi$ be “ $x_{1}=x_{2}$, " $j^{*}$ is well defined; replacing $\phi$ by $\neg \phi, j^{*}$ is fully elementary.

Fact 2. Let $M, N, j, P \in M$ be as in the statement of Fact 1 . Let $j$ be generated by an extender with width $\mu$, and let $M \vDash$ " $P$ is $\mu^{+}$-dense." Let $G$ be $P$-generic over $M$. Then

$$
H=\{q \in j(P) \mid \exists p \in G j(p) \leq q\}
$$


is $j(P)$-generic over $N$, and as $(*)$ holds $j$ extends to an elementary embedding $j^{*}: M[G] \rightarrow N[H]$.

Proof. Let $D \in N, D \subseteq j(P)$ be a dense open subset. $D=j(f)(a)$, where $a \in[\mathrm{ON}]^{<\omega}, f \in M$, and

$\forall x \in[\mu]^{|a|} f(x)$ is a dense open subset of $P$.

As $M \vDash P \mu^{+}$-dense,

$$
D^{*}=\bigcap_{x \in[\mu]^{|a|}} f(x)
$$

is a dense open subset of $P . D^{*} \in M$, so find $p$ such that $p \in D^{*} \cap G$. $M \vDash \forall x \quad p \in f(x)$, thus $N \vDash j(p) \in j(f)(a)=D$. So $D \cap H \neq \varnothing$, hence $H$ is generic.

Fact 3. Let $M, N, j, P, G, H$ be as in the statement of Fact 1. Let (*) hold, and let $j$ be generated by a $(\kappa, \lambda)$-M-extender. Then $j^{*}$ is so generated. Proof. Let $x \in N[H] . \quad x=i_{H}(\dot{\tau})$ for some $\dot{\tau} \in N^{j(P)} . \dot{\tau}=j(f)(a)$, where $a \in[\lambda]^{<\omega}, f \in M, f:[\mu]^{|a|} \rightarrow M^{P}$. Define $f^{*} \in M[G], \operatorname{dom}\left(f^{*}\right)=[\mu]^{|a|}$, $f^{*}: y \mapsto i_{G}(f(y))$. Clearly $x=j^{*}\left(f^{*}\right)(a)$, so

$$
N[H]=\left\{j^{*}(g)(b) \mid g \in M[G], b \in[\lambda]^{<\omega}\right\} .
$$

3. Building generics. We collect some facts pertinent to constructing generics over one model inside another.

Fact 1. Let $M, N$ be inner models of $Z F C, M \subseteq N$. Let $P \in M$,

$N \vDash$ " $P$ is $\lambda$-closed,"

$N \vDash \mid\{A \mid A$ is a maximal antichain in $P, A \in M\} \mid \leq \lambda$.

Then there is in $N$ a P-generic filter over $M$.

Proof. In $N$, enumerate the set of maximal antichains of $P$ lying in $M$ as $\left\langle A_{\alpha}: \alpha<\lambda\right\rangle$. Build by induction a sequence $\left\langle p_{\alpha}: \alpha<\lambda\right\rangle$ such that

$$
\alpha<\beta \Rightarrow p_{\beta} \leq p_{\alpha}, \quad \forall \alpha \exists x \in A_{\alpha} \quad p_{\alpha} \leq x .
$$

The closure enables the construction to be continued at limit stages.

$$
G=\left\{p \in P \mid \exists \alpha p_{\alpha} \leq p\right\}
$$

is clearly a filter on $P$ which is generic over $M$.

Fact 2. Let $M, N$ be inner models of $Z F C, M \subseteq N, \kappa \in \mathrm{ON}$. Then

$$
N \vDash^{\kappa} M \subseteq M \Leftrightarrow N \vDash^{\kappa} \mathrm{ON} \subseteq M .
$$

Proof. By AC, every set in $M$ is in bijection with some ordinal.

Fact 3. Let $M, N$ be inner models of ZFC, $M \subseteq N, N \vDash{ }^{\kappa} M \subseteq M$. Let $P \in M$ be a forcing notion, $N \vDash$ " $P$ is $\kappa^{+}$-c.c." and let $G$ be $P$-generic over $N$. Then $N[G] \vDash{ }^{\kappa} M[G] \subseteq M[G]$.

Proof. By Fact 2 it is enough to show that $N[G] \vDash{ }^{\kappa} \mathrm{ON} \subseteq M[G]$. But it is immediate from the chain condition of $P$ in $N$ that to any name in $N^{P}$ for a $\kappa$-sequence of ordinals there corresponds an equivalent name in $M^{P}$.

4. Mutual genericity. We give some results which guarantee the mutual genericity of generic objects under certain circumstances. 
Fact 1 (Easton's Lemma). Let $P$ a $\kappa$-c.c notion of forcing, and $Q$ be a $\kappa$-closed one. Then $\Vdash_{P}$ " $\hat{Q}$ is $\hat{\kappa}$-dense," $\Vdash_{Q}$ " $\hat{P}$ is $\hat{\kappa}$-c.c," and generics for $P$ and $Q$ are mutually generic.

Proof. Let $\dot{A} \in V^{Q}, q \in Q$, be such that

$$
q \Vdash \text { “ } \dot{A}: \hat{\kappa} \rightarrow \hat{P} \text { enumerates an antichain." }
$$

Define a descending sequence $\left\langle q_{\alpha}: \alpha<\kappa\right\rangle$ of elements of $Q$, and a sequence $\left\langle p_{\alpha}: \alpha<\kappa\right\rangle$ of elements of $P$ such that $q_{0}=q$ and $\forall \alpha<\kappa q_{\alpha+1} \Vdash \dot{A}(\hat{\alpha})=\hat{p}_{\alpha}$. Then clearly $\left\langle p_{\alpha}: \alpha<\kappa\right\rangle$ enumerates an antichain in $P$ lying in $V$; this is a contradiction, so we conclude that $\Vdash_{Q} \hat{P} \hat{\kappa}$-c.c.

Let $G$ be $P$-generic, and $H$ be $Q$-generic. The mutual genericity of $G$ and $H$ is immediate, for if $A \in V[H]$ is a maximal antichain in $P$ then $|A|<\kappa$ so $A \in V$ and $A \cap G \neq \varnothing$.

Finally we show that $V[G]$ and $V[G][H]$ have the same $<\kappa$-sequences of ordinals. $V[G][H]=V[H][G]$, so let $\dot{\tau} \in V[H]^{P}$ be a term for such a sequence. By a chain condition argument we can assume $\dot{\tau} \subset P \times \mathrm{ON}$ and $\| \dot{\tau}<\kappa$, so $\dot{\tau} \in V$ by closure.

The following fact is due to Woodin, and plays a central role in [W].

Fact 2. Let $\kappa$ be a measurable cardinal, $U$ some normal measure on $\kappa$, and $j: V \rightarrow M$ the ultrapower by $U$. Let $P$ be a $\kappa$-closed partial ordering, and let $Q=j(P) .{ }^{\kappa} M \subseteq M$ and $j(\kappa)>\kappa$, so $Q$ is $\kappa^{+}$-closed; let $G$ be $Q$-generic over $V$, and transfer it along $j$ to get $j^{*}: V[G] \rightarrow M\left[j^{*}(G)\right]$. Then $G$ is in fact $Q$-generic over $M\left[j^{*}(G)\right]$.

Proof. Let $\dot{D} \in M^{j(Q)}$ be a name for a dense subset of $Q$. As $M$ is an ultrapower, $\dot{D}$ is of the form $j(f)(\kappa)$, where for each $\alpha \quad f(\alpha) \in V^{Q}$ is a name for a dense subset of $P$. Define a subset of $Q$ by

$$
E=\{q \in Q \mid \exists g: \kappa \rightarrow P \quad q=j(g)(\kappa) \wedge \forall \alpha q \Vdash \widehat{g(\alpha)} \in f(\alpha)\} .
$$

The claim is that $E$ is a dense subset of $Q$. Fix $r$ in $Q$, and let $r$ be represented as $j(h)(\kappa)$ for some $h: \kappa \rightarrow P$. Construct by induction on $\alpha<\kappa$ functions $h_{\alpha}$ and elements of $P p_{\alpha}$ such that

$$
\begin{aligned}
& h_{\alpha}: \kappa \rightarrow P, \text { and if } \alpha<\beta \text { then } \forall \gamma h_{\beta}(\gamma) \leq_{P} h_{\alpha}(\gamma), \\
& j\left(h_{\alpha}\right)(\kappa) \Vdash \widehat{p_{\alpha}} \in f(\alpha), \\
& \forall \gamma \leq \alpha h_{\alpha}(\gamma)=p_{\gamma}, \\
& j\left(h_{0}\right)(\kappa) \leq r .
\end{aligned}
$$

Construction. At stage $\alpha$, let $p \in P$ be some lower bound for the sequence $\left\langle h_{\beta}(\alpha): \beta<\alpha\right\rangle$, and let $q \in Q$ be a lower bound for the sequence $\left\langle j\left(h_{\beta}\right)(\kappa)\right.$ : $\beta<\alpha\rangle . f(\alpha)$ names a dense subset of $P$, so find $q^{*} \leq q, p^{*} \leq p$, such that $q^{*} \Vdash \widehat{p^{*}} \in f(\alpha)$. Let $p_{\alpha}$ be $p^{*}$, and alter some function representing $q^{*}$ to get an appropriate $h_{\alpha}$. (This is possible because we only need alter the function on a set of measure zero.) Define $h^{*}$ by $h^{*}(\alpha)=p_{\alpha}$; then $r^{*}={ }_{\operatorname{def}} j\left(h^{*}\right)(\kappa)$ is an extension of $r$ lying in $E$.

$\begin{array}{lllll}h(0) & h(1) & \ldots & h(\alpha) & \ldots \\ p_{0} & h_{0}(1) & \ldots & h_{0}(\alpha) & \ldots \\ p_{0} & p_{1} & \ldots & h_{1}(\alpha) & \ldots \\ \vdots & \vdots & \ddots & \vdots & \ldots \\ p_{0} & p_{1} & \ldots & p_{\alpha} & \ldots\end{array}$


As $E$ lies in $V$ and is dense, find $q=j(h)(\kappa)$ such that $q \in E \cap G$, where $h$ is chosen to witness $q \in E . q \Vdash \forall \alpha<\kappa \widehat{h(\alpha)} \in f(\alpha)$, so applying $j$ and letting $\alpha$ be $\kappa, j(q) \Vdash j(\widehat{h)(} \kappa) \in j(f)(\kappa) . j(q) \in j^{*}(G)$, so $q \in i_{j^{*}(G)}(\dot{D})$ hence $G$ is $Q$-generic over $M\left[j^{*}(G)\right]$.

5. Term forcing. We describe a forcing trick which is needed for the Reverse Easton construction of $\S 2$.

Let $P$ be a forcing notion and $\dot{Q} \in V^{P}$; then $\Vdash_{P} \dot{Q}$ is a forcing notion. Define a forcing $\dot{Q} / P \in V$ by

$$
\begin{gathered}
\dot{\tau} \in \dot{Q} / P \Leftrightarrow \dot{\tau} \in V^{P} \wedge \Vdash_{P} \dot{\tau} \in \dot{Q}, \\
\dot{\tau} \leq_{\dot{Q} / P} \dot{\sigma} \Leftrightarrow \Vdash_{P} \dot{\tau} \leq_{\dot{Q}} \dot{\sigma} .
\end{gathered}
$$

$\dot{Q} / P$ is technically a class, but can be treated as a set, as in the standard treatment of iterated forcing . (see $[\mathrm{B}]$ or $[\mathrm{K}]$ ).

Forcing with $\dot{Q} / P$ adds a "universal generic object" for $\dot{Q}$. More precisely we have

Fact 1. Let $H$ be $\dot{Q} / P$-generic over $V$, and let $G$ be $P$-generic over $V$. Then $F=\left\{i_{G}(\dot{\tau}) \mid \dot{\tau} \in H\right\}$ is $i_{G}(\dot{Q})$-generic over $V[G]$.

Proof.

Claim 1. $F$ is a filter.

Proof of Claim 1.

$$
\begin{aligned}
& \dot{\rho}, \dot{\sigma} \in H \Rightarrow \exists \dot{\tau} \in H \quad \Vdash_{P} \dot{\tau} \leq_{\dot{Q}} \dot{\rho}, \dot{\sigma} \Rightarrow i_{G}(\dot{\tau}) \leq_{i_{G}(\dot{Q})} i_{G}(\dot{\rho}), i_{G}(\dot{\sigma}), \\
& \dot{\rho} \in H \wedge i_{G}(\dot{\rho}) \leq i_{G}(\dot{\sigma}) \Rightarrow \exists \dot{\tau} \quad i_{G}(\dot{\tau})=i_{G}(\dot{\sigma}) \wedge \Vdash \dot{\rho} \leq \dot{\tau} \Rightarrow \dot{\tau} \in H
\end{aligned}
$$

Claim 2. $F$ is generic.

Proof of Claim 2. Let $\dot{D}$ be a name for a dense subset of $\dot{Q}$, and define

$$
E=\left\{\dot{\tau} \in \dot{Q} / P \mid \Vdash_{P} \dot{\tau} \in \dot{D}\right\} \text {. }
$$

$E$ is dense in $\dot{Q} / P$; for if $\dot{\rho} \in \dot{Q} / P$,

$$
\Vdash_{P} \exists x \quad x \in \dot{D} \wedge x \leq \dot{\rho},
$$

so by the maximum principle there is $\dot{\tau}$ such that

$$
\Vdash_{P} \dot{\tau} \in \dot{D} \wedge \dot{\tau} \leq \dot{\rho}
$$

As $H$ is generic and $E \in V$, for some $\dot{\tau} \in H \quad i_{G}(\dot{\tau}) \in i_{G}(\dot{D})$.

The proof of Fact 1 is complete.

There are a couple of interesting cases in which, if $Q$ is some forcing notion defined in the ground model and $\dot{Q}$ names the forcing defined by working that definition in $V^{P}$, then $Q$ is isomorphic to $\dot{Q} / P$. This is useful in Reverse Easton forcing as another way of transferring generics between models. 
Fact 2. Let $P$ be a forcing notion of cardinality $\kappa$ with the $\kappa-c . c$. , where $\kappa$ is such that $\kappa^{<\kappa}=\kappa$. Then

(a) For any $\lambda \operatorname{Add}(\kappa, \lambda)_{V^{P}} / P$ is isomorphic to $\operatorname{Add}(\kappa, \lambda)$.

(b) If $\lambda \geq \kappa$ is such that $\lambda^{<\kappa}=\lambda$ then $\operatorname{Coll}(\kappa, \lambda)_{V^{P}} / P$ is isomorphic to $\operatorname{Coll}(\kappa, \lambda)$.

Proof. If $\beta$ is some cardinal let $P_{\beta}$ denote the partial ordering which has a top element $T$ with $\beta$ incomparable extensions (which we identify with the elements of $\beta$ ).

For (a), recall that $A d d(\kappa, \lambda)$ is the product of $\lambda$ copies of $P_{2}$ with $<\kappa$ support. As $P$ is $\kappa$-c.c. it is clear that $\operatorname{Add}(\kappa, \lambda)_{V^{P}} / P$ is just the product of $\lambda$ copies of $P_{2} / P$ with $<\kappa$-support. If $i \in P_{2} / P, i$ is determined up to equivalence by the pair of Boolean values $([\dot{\tau}=0],[\dot{\tau}=1])$, and any pair $\left(b_{1}, b_{2}\right)$ such that $b_{1} \wedge b_{2}=0$ corresponds to a name: moreover the ordering is just the restriction of the product order on $R . O .(P) \times R . O .(P)$. This ordering has a dense subset isomorphic with $P_{\kappa}$, where the $\kappa$ incomparable elements are the pairs $(b, \neg b)$ for $b \in R . O .(P)$.

So $\operatorname{Add}(\kappa, \lambda)_{V^{P}} / P$ is isomorphic to the product of $\lambda$ copies of $P_{\kappa}$ with $<\kappa$-support, i.e., isomorphic to $\operatorname{Add}(\kappa, \lambda)$ as claimed. The proof for (b) is exactly similar with $P_{\lambda}$ playing the role of $P_{2} ; \lambda^{<\kappa}=\lambda$ guarantees that $P_{\lambda} / P$ is isomorphic to $P_{\lambda}$.

6. Two facts about Cohen forcing. The following facts are also needed in $\S 2$.

Fact 1. Let $\kappa=\operatorname{cf}(\kappa)>\omega$ and let $P$ be a $\kappa$-closed notion of forcing with $|P|=\kappa$. Then $P$ is isomorphic to the Cohen forcing $\operatorname{Add}(\kappa, 1)$.

Proof. The proof is just like the proof (see [J, p. 277]) that a forcing which collapses $\lambda$ and has size $\lambda$ is isomorphic to $\operatorname{Coll}(\omega, \lambda)$.

Fact 2. Let $j: V \rightarrow M$ be the ultrapower of $V$ by a normal measure $U$ on some cardinal $\kappa$, where $2^{\kappa}=\kappa^{+}$. Then $j\left(\operatorname{Add}\left(\kappa, \kappa^{++}\right)\right)$is isomorphic in $V$ to $\operatorname{Add}\left(\kappa^{+}, \kappa^{++}\right)$.

Proof. As $2^{\kappa}=\kappa^{+}$and $j$ is an ultrapower embedding, $j\left(\kappa^{++}\right)=\kappa^{++}$and the set $S$ defined by

$$
S=\left\{\alpha<\kappa^{++} \mid j(\alpha)=\alpha\right\}
$$

is unbounded in $\kappa^{++}$. Let $\bar{S}$, the closure of the set $S$, be enumerated in increasing order as $\left\langle\lambda_{\nu}: \nu<\kappa^{++}\right\rangle$. As $\bar{S}$ is club in $\kappa^{++}$,

$$
\kappa^{++}=\bigcup_{\nu<\kappa^{++}}\left[\lambda_{\nu}, \lambda_{\nu+1}\right)
$$

In $V$ the forcing $\operatorname{Add}\left(j(\kappa),\left[\lambda_{\nu}, \lambda_{\nu+1}\right)\right)_{M}$ is $\kappa^{+}$-closed and of cardinality $\kappa^{+}$, hence isomorphic by Fact 1 to the forcing $\operatorname{Ad} d\left(\kappa^{+},\left[\lambda_{\nu}, \lambda_{\nu+1}\right)\right)$. Choose for each $\nu$ an isomorphism $\pi_{\nu}$, and define a function $\pi$ with the domain $\operatorname{Add}\left(j(\kappa), j\left(\kappa^{++}\right)\right)_{M}$ by

$$
\pi: p \mapsto \bigcup_{\nu<\kappa^{++}} \pi_{\nu}\left(p\left\lceil j(\kappa) \times\left[\lambda_{\nu}, \lambda_{\nu+1}\right)\right) .\right.
$$

Then we claim $\pi$ is a bijection onto $\operatorname{Add}\left(\kappa^{+}, \kappa^{++}\right)$. 
First we show that $\pi$ maps into $\operatorname{Ad} d\left(\kappa^{+}, \kappa^{++}\right)$. Let $p$ be a condition in $\operatorname{Ad} d\left(j(\kappa), \kappa^{++}\right)_{M}$. It is necessary to show that $\pi(p)$ is a condition in $\operatorname{Add}\left(\kappa^{+}, \kappa^{++}\right)$, which will be immediate once we check that the support of $\pi(p)$ has cardinality $\leq \kappa . \exists \nu<\kappa^{++} \operatorname{support}(p) \subseteq j(\kappa) \times \lambda_{\nu}$, and therefore $\operatorname{support}(\pi(p)) \subseteq \kappa^{+} \times \lambda_{\nu}$. Do an induction on $\mu \leq \nu$ with the hypothesis that $\pi(p)\left\lceil\kappa^{+} \times \lambda_{\mu}\right.$ has support of cardinality $\leq \kappa$. The induction step is trivial at successor $\mu$ and those limit $\mu$ with $\operatorname{cf}_{V}(\mu) \leq \kappa$. So assume $\operatorname{cf}_{V}(\mu)>\kappa$; $\lambda_{\mu} \in S$, because $\operatorname{cf}_{V}\left(\lambda_{\mu}\right)=\operatorname{cf}_{V}(\mu)>\kappa$ and so $\lambda_{\mu}$ is a continuity point of $j$. So

$$
\operatorname{cf}_{M}\left(\lambda_{\mu}\right)=\operatorname{cf}_{M}\left(j\left(\lambda_{\mu}\right)\right)=j\left(\operatorname{cf}_{V}\left(\lambda_{\mu}\right)\right)>j(\kappa) .
$$

Hence $\exists \rho<\mu$ support $\left(p\left\lceil j(\kappa) \times \lambda_{\mu}\right) \subseteq j(\kappa) \times \lambda_{\rho}\right.$ and the induction hypothesis goes through.

To show that $\pi$ is onto, let $q \in \operatorname{Add}\left(\kappa^{+}, \kappa^{++}\right)$. Because ${ }^{\kappa} M \subset M$, and $q$ has support of cardinality $\leq \kappa$, the sequence

$$
\left\langle\pi_{\nu}^{-1}\left(q\left\lceil\kappa^{+} \times\left[\lambda_{\nu}, \lambda_{\nu+1}\right)\right): \nu<\kappa^{++} \wedge \operatorname{support}(q) \cap \kappa^{+} \times\left[\lambda_{\nu}, \lambda_{\nu+1}\right) \neq \varnothing\right\rangle\right.
$$

is in $M$. As $\kappa<j(\kappa)$ and $j(\kappa)$ is regular in $M$, the union of this sequence is a condition which maps to $q$ under $\pi$.

1.3. The structure of the proof. As the proof is rather long and complicated we give a brief overview of what is going on.

We start with the hypotheses

(1) $\kappa$ is $\mathscr{P}_{3} \kappa$-hypermeasurable, as witnessed by $j: V \rightarrow M$ with ${ }^{\kappa} M \subset M$, $V_{\kappa+3} \subset M, \operatorname{crit}(j)=\kappa$.

(2) $\mathrm{GCH}$.

(3) $j$ is generated by a $\left(\kappa, \kappa^{+3}\right)$-extender.

(3) is really superfluous as given a model of (1), (2) we can factor through an extender to get (3).

In $\S 2$ we do two Reverse Easton extensions, and at the end we have a model of the hypotheses

(1) $\kappa$ is $\mathscr{P}_{2} \kappa$-hypermeasurable, as witnessed by $j: V \rightarrow M$ with ${ }^{\kappa} M \subset M$, $V_{\kappa+2} \subset M, \operatorname{crit}(j)=\kappa$.

(2) For each strong inaccessible $\alpha \leq \kappa, 2^{\alpha}=\alpha^{++}$and, for all $n$, $0<n<\omega \Rightarrow 2^{\alpha^{+n}}=\alpha^{+n+1}$.

(3) $M \vDash 2^{\kappa}=\kappa^{++} \wedge \forall n \quad 0<n<\omega \Rightarrow 2^{\kappa^{+n}}=\kappa^{+n+1}$.

(4) There is an $F \in V$ which is $P$-generic over $N$, where

$$
P=\operatorname{Coll}\left(\kappa^{+4},<i(\kappa)\right) \times \operatorname{Coll}\left(i(\kappa), i\left(\kappa^{+}\right)\right)_{N}
$$

and $i: V \rightarrow N$ is the ultrapower of $V$ by the ultrafilter

$$
U=\{X \subset \kappa \mid \kappa \in j(X)\} .
$$

(5) $j$ is generated by a $\left(\kappa, \kappa^{+3}\right)$-extender.

Then in $\S 3$ we use these hypotheses to define a modified Radin forcing; this forcing will shoot a closed unbounded set of $V$-measurables $C$ through $\kappa$, and in addition if $\alpha, \beta$ are successive points of $C$ it will add a generic for the collapsing algebra $\operatorname{Coll}\left(\alpha^{+4},<\beta\right) \times \operatorname{Coll}\left(\beta, \beta^{+}\right)$. 
We prove analogues of the Prikry Lemma and Mathias' criterion for Prikry genericity, and use these to show that the forcing has produced the right behaviour for the continuum function below $\kappa$ and that $\kappa$ has retained its hypermeasurability.

\section{PREPARING THE MODEL}

2.1. First stage. Recall that the initial hypotheses are

(1) $\kappa$ is $\mathscr{P}_{3} \kappa$-hypermeasurable, as witnessed by $j: V \rightarrow M$ with ${ }^{\kappa} M \subset M$, $V_{\kappa+3} \subset M, \operatorname{crit}(j)=\kappa$.

(2) $\mathrm{GCH}$.

(3) $j$ is generated by a $\left(\kappa, \kappa^{+3}\right)$-extender.

Factor $j$ as $k \circ i$, where $i: V \rightarrow N$ is the ultrapower by the ultrafilter $U=\{X \subseteq \kappa \mid \kappa \in j(X)\}$ and $k:[f]_{U}=i(f)(\kappa) \mapsto j(f)(\kappa)$. Let $\lambda$ be the critical point of $k$. GCH implies that $\lambda=\kappa_{N}^{++}$, and $\kappa^{+}<\lambda<\kappa^{++}$. As we remarked in the introduction $k$ is generated by a $\left(\lambda, \kappa^{+3}\right)-N$-extender of width $\kappa_{N}^{+3}$.

We do the Reverse Easton iteration of $\operatorname{Add}\left(\alpha^{+}, \alpha^{++}\right)$for strong inaccessible $\alpha \leq \kappa$. Let $P_{\kappa}$ denote the forcing up to stage $\kappa$, and let $G_{\kappa}$ be $P_{\kappa}$-generic over $V$. Let $Q_{\kappa}=\left(\operatorname{Add}\left(\kappa^{+}, \kappa^{++}\right)\right)_{V\left[G_{\kappa}\right]}$ be the forcing we do at stage $\kappa$, and let $g$ be $Q_{\kappa}$-generic over $V\left[G_{\kappa}\right]$. Factor $\operatorname{Add}\left(\kappa^{+}, \kappa^{++}\right)$as

$$
\left(\operatorname{Add}\left(\kappa^{+}, \lambda\right) \times \operatorname{Add}\left(\kappa^{+}, \kappa^{++} \backslash \lambda\right)\right)_{V\left[G_{\kappa}\right]}
$$

and split up $g$ as $g_{1} \times g_{2}$ correspondingly.

Now consider the iterations $j\left(P_{\kappa} * \dot{Q}_{\kappa}\right)$ and $i\left(P_{\kappa} * \dot{Q}_{\kappa}\right)$ computed by $M$ and $N$ respectively. As the three models $V, M, N$ agree to rank $\kappa$ they compute the same iteration below $\kappa$, so defining $\left(P_{\kappa}\right)_{M}$ and $\left(P_{\kappa}\right)_{N}$ in the obvious way,

$$
P_{\kappa}=\left(P_{\kappa}\right)_{M}=\left(P_{\kappa}\right)_{N} \text {, }
$$

$P_{\kappa}$ is $\kappa$-c.c. and therefore

$$
V\left[G_{\kappa}\right] \vDash{ }^{\kappa} M\left[G_{\kappa}\right] \subset M\left[G_{\kappa}\right], \quad V\left[G_{\kappa}\right] \vDash{ }^{\kappa} N\left[G_{\kappa}\right] \subset N\left[G_{\kappa}\right] .
$$

So $\kappa^{+}=\kappa_{V\left[G_{\kappa}\right]}^{+}=\kappa_{M\left[G_{\kappa}\right]}^{+}=\kappa_{N\left[G_{\kappa}\right]}^{+}$. Because $V_{\kappa+2} \subset M$ we have $\kappa^{++}=\kappa_{M}^{++}=$ $\kappa_{M\left[G_{\kappa}\right]}^{++}$, and so if $\left(Q_{\kappa}\right)_{M}$ is the forcing $M\left[G_{\kappa}\right]$ computes at $\kappa$ then

$$
\left(Q_{\kappa}\right)_{M}=\left(\operatorname{Add}\left(\kappa^{+}, \kappa^{++}\right)\right)_{M\left[G_{\kappa}\right]}=\left(\operatorname{Add}\left(\kappa^{+}, \kappa^{++}\right)\right)_{V\left[G_{\kappa}\right]}=Q_{\kappa} .
$$

On the other hand if $\left(Q_{\kappa}\right)_{N}$ is the forcing which $N\left[G_{\kappa}\right]$ computes at $\kappa$ then because $\kappa_{N\left[G_{\kappa}\right]}^{++}=\kappa_{N}^{++}=\lambda$ we have

$$
\left(Q_{\kappa}\right)_{N}=\left(\operatorname{Add}\left(\kappa^{+}, \kappa^{++}\right)\right)_{N\left[G_{\kappa}\right]}=\left(\operatorname{Add}\left(\kappa^{+}, \lambda\right)\right)_{V\left[G_{\kappa}\right]}
$$

Now $k\left(P_{\kappa}\right)=P_{\kappa}$ and $\forall p \in G_{\kappa} k(p)=p$, so we can extend $k$ (using Fact $1, \S 1.2 .3)$ to

$$
k: N\left[G_{\kappa}\right] \rightarrow M\left[G_{\kappa}\right] .
$$

$k\left(\left(Q_{\kappa}\right)_{N}\right)=\left(Q_{\kappa}\right)_{M}=Q_{\kappa}$, and since $\operatorname{crit}(k)=\lambda$ and $g_{1} \subset g$ we have that $\forall p \in g_{1} \quad k(p)=p \in g$. So we can extend $k$ further to get

$$
k: N\left[G_{\kappa}\right]\left[g_{1}\right] \rightarrow M\left[G_{\kappa}\right][g] .
$$

Because the forcing notion $\left(Q_{\kappa}\right)_{N}$ is $\kappa^{+}$closed it adds no $\kappa$-sequences of ordinals, so

$$
V\left[G_{\kappa}\right]\left[g_{1}\right] \vDash{ }^{\kappa} N\left[G_{\kappa}\right]\left[g_{1}\right] \subset N\left[G_{\kappa}\right]\left[g_{1}\right] .
$$


Also, because $N$ is an ultrapower and $\mathrm{GCH}$ holds in $V$,

$$
V\left[G_{\kappa}\right]\left[g_{1}\right] \vDash|i(\kappa)|=\kappa^{+} .
$$

Consider the iteration $R$ which $N\left[G_{\kappa}\right]\left[g_{1}\right]$ does between $\kappa+1$ and $i(\kappa)$. Standard facts about Reverse Easton forcing imply that

$$
N\left[G_{\kappa}\right]\left[g_{1}\right] \vDash|R|=i(\kappa), R \text { is } i(\kappa) \text {-c.c. and } \kappa^{+4} \text {-closed. }
$$

We exploit these facts to build $H \in V\left[G_{\kappa}\right]\left[g_{1}\right]$ which is $R$-generic over $N\left[G_{\kappa}\right]\left[g_{1}\right]$. The closure of $R$ in $N\left[G_{\kappa}\right]\left[g_{1}\right]$ and the width of the extender for $k$ allow us to transfer $H$ along $k$, and get $k(H) k(R)$-generic over $M\left[G_{\kappa}\right][g]$.

So we can build maps

$$
\begin{aligned}
& i: V\left[G_{\kappa}\right] \rightarrow N\left[G_{\kappa}\right]\left[g_{1}\right][H], \\
& j: V\left[G_{\kappa}\right] \rightarrow M\left[G_{\kappa}\right][g][k(H)], \\
& k: N\left[G_{\kappa}\right]\left[g_{1}\right][H] \rightarrow M\left[G_{\kappa}\right][g][k(H)]
\end{aligned}
$$

such that $j=k \circ i$.

The map $i: V\left[G_{\kappa}\right] \rightarrow N\left[G_{\kappa}\right]\left[g_{1}\right][H]$ is defined in $V\left[G_{\kappa}\right]\left[g_{1}\right]$. Since $Q_{\kappa}$ is $\kappa^{+}$-closed and $i$ is generated by an extender of width $\kappa$ we can transfer $g_{1}$ along $i$ and get an internal ultrapower

$$
i: V\left[G_{\kappa}\right]\left[g_{1}\right] \rightarrow N\left[G_{\kappa}\right]\left[g_{1}\right][H]\left[i\left(g_{1}\right)\right]=N\left[i\left(G_{\kappa} * g_{1}\right)\right] .
$$

Now let $Q_{1}={ }_{\text {def }}\left(\operatorname{Add}\left(\kappa, \kappa^{++}\right)\right)_{V\left[G_{\kappa}\right]\left[g_{1}\right]}$. As $V\left[G_{\kappa}\right]\left[g_{1}\right]$ is a model of $2^{\kappa}=\kappa^{+}$ Fact I.2.6.2 implies that in $V\left[G_{\kappa}\right]\left[g_{1}\right]$

$$
i\left(Q_{1}\right) \simeq \operatorname{Add}\left(\kappa^{+}, \kappa^{++}\right) .
$$

Let $Q_{2}=_{\text {def }}\left(\operatorname{Coll}\left(\kappa, \kappa^{+}\right)\right)_{V\left[G_{\kappa}\right]\left[g_{1}\right]}$. In $V\left[G_{\kappa}\right]\left[g_{1}\right]$ we also know that $\left|i\left(\kappa^{+}\right)\right|$ $=\kappa^{+}$and $i\left(Q_{2}\right)$ is $\kappa^{+}$-closed so

$$
i\left(Q_{2}\right) \simeq \operatorname{Add}\left(\kappa^{+}, 1\right) .
$$

The upshot of all this is that in $V\left[G_{\kappa}\right]\left[g_{1}\right]$

$$
i\left(Q_{1} \times Q_{2}\right) \simeq \operatorname{Add}\left(\kappa^{+}, \kappa^{++} \backslash \lambda\right) .
$$

Recall that $g_{2}$ is generic over $V\left[G_{\kappa}\right]\left[g_{1}\right]$ for this latter forcing (by closure, this has the same definition in $V\left[G_{\kappa}\right]$ and $\left.V\left[G_{\kappa}\right]\left[g_{1}\right]\right)$ so letting

$$
Q==_{\text {def }} i\left(Q_{1} \times Q_{2}\right)
$$

we can rearrange $g_{2}$ as $\bar{g}$ a $Q$-generic over $V\left[G_{\kappa}\right]\left[g_{1}\right]$.

Transferring $g_{2}$ along $i, j$ we get maps

$$
\begin{aligned}
& i: V\left[G_{\kappa}\right][g] \rightarrow N\left[i\left(G_{\kappa} * g\right)\right], \\
& j: V\left[G_{\kappa}\right][g] \rightarrow M\left[j\left(G_{\kappa} * g\right)\right], \\
& k: N\left[i\left(G_{\kappa} * g\right)\right] \rightarrow M\left[j\left(G_{\kappa} * g\right)\right]
\end{aligned}
$$

with $j=k \circ i$.

Let $V^{*}=V\left[G_{\kappa}\right][g], N^{*}=N\left[i\left(G_{\kappa} * g\right)\right], M^{*}=M\left[j\left(G_{\kappa} * g\right)\right]$. From Fact 2, $\S 1.2 .4$, we have that $\bar{g}$ is $Q$-generic over $N^{*}$. Closure implies that

$$
Q_{1} \times Q_{2}=\left(\operatorname{Add}\left(\kappa, \kappa^{++}\right) \times \operatorname{Coll}\left(\kappa, \kappa^{+}\right)\right)_{V^{*}}
$$


and hence that

$$
Q=\left(\operatorname{Add}\left(i(\kappa), i\left(\kappa^{++}\right)\right) \times \operatorname{Coll}\left(i(\kappa), i\left(\kappa^{+}\right)\right)\right)_{N^{*}} .
$$

It is also worth observing that $i: V^{*} \rightarrow N^{*}$ is the ultrapower of $V^{*}$ by the normal measure on $\kappa$ induced by $j$.

We claim that $j: V^{*} \rightarrow M^{*}$ is a $\mathscr{P}_{3} \kappa$-hypermeasurable embedding. As GCH holds in $V^{*}$ it is enough to show that

$$
V^{*} \vDash \mathscr{P}\left(\kappa^{++}\right) \subset M
$$

This follows immediately from the fact that the forcing $P_{\kappa} * \dot{Q}_{\kappa}$ has size $\kappa^{++}$ and is $\kappa^{++}$-c.c., so that every subset of $\kappa^{++}$in $V^{*}$ has a canonical name in $M$.

We now have a model of the hypotheses

(1) $\kappa$ is $\mathscr{P}_{3} \kappa$-hypermeasurable, as witnessed by $j: V \rightarrow M$ with ${ }^{\kappa} M \subset M$, $V_{\kappa+3} \subset M, \operatorname{crit}(j)=\kappa$.

(2) $\mathrm{GCH}$.

(3) $j$ is generated by a $\left(\kappa, \kappa^{+3}\right)$-extender.

(4) There is in $V$ an object $\bar{g}$ which is $i(P)$-generic over $N$, where $P=\operatorname{Add}\left(\kappa, \kappa^{++}\right) \times \operatorname{Coll}\left(\kappa, \kappa^{+}\right)$and $i: V \rightarrow N$ is the ultrapower by $\{X \subseteq \kappa \mid \kappa \in j(X)\}$.

2.2. Second stage. We are given the hypotheses from the end of the first stage. Factor $j$ in two steps through the models

$$
\begin{aligned}
& N=\text { the collapse of }\{j(f)(\kappa) \mid f: \kappa \rightarrow V\}, \\
& \bar{N}=\text { the collapse of }\left\{j(f)(a) \mid a \in\left[\kappa^{++}\right]^{<\omega} \wedge f:[\kappa]^{|a|} \rightarrow V\right\} .
\end{aligned}
$$

$N$ is the familiar ultrapower approximating $M$, while $\bar{N}$ corresponds to the extender of length $\kappa^{++}$. We have maps

$$
\begin{array}{ll}
i: V \rightarrow N, & k: N \rightarrow M, \\
\bar{i}: N \rightarrow \bar{N}, & \bar{k}: \bar{N} \rightarrow M
\end{array}
$$

such that

$$
j=k \circ i, \quad k=\bar{k} \circ \bar{i}
$$

Let $\lambda=\kappa_{N}^{++}$, and argue as before that $\kappa^{+}<\lambda<\kappa^{++}$.

Do the reverse Easton iteration where we force at inaccessible $\lambda \leq \kappa$ with $\operatorname{Add}\left(\lambda, \lambda^{++}\right)$. Let $P_{\kappa}$ be the forcing up to $\kappa$, and let $G_{\kappa}$ be $P_{\kappa}$-generic over $V$. Let

$$
\begin{aligned}
Q_{\kappa} & =\left(\operatorname{Add}\left(\kappa, \kappa^{++}\right)\right)_{V\left[G_{\kappa}\right]}, \\
P & =\left(\operatorname{Add}\left(\kappa, \kappa^{++}\right) \times \operatorname{Coll}\left(\kappa, \kappa^{+}\right)\right)_{V} .
\end{aligned}
$$

Let $g$ be $Q_{\kappa}$-generic over $V\left[G_{\kappa}\right]$, and let $\bar{g}$ be $i(P)$-generic over $N$ as guaranteed by the hypotheses.

The same argument as in stage one shows that all four models compute the same forcing up to $\kappa$, and it is easy to extend the maps $k, \bar{i}, \bar{k}$ to get

$$
k: N\left[G_{\kappa}\right] \rightarrow M\left[G_{\kappa}\right], \quad \bar{i}: N\left[G_{\kappa}\right] \rightarrow \bar{N}\left[G_{\kappa}\right], \quad \bar{k}: \bar{N}\left[G_{\kappa}\right] \rightarrow M\left[G_{\kappa}\right],
$$

where $k=\bar{k} \circ \bar{i}$. 
$\bar{N}\left[G_{\kappa}\right], M\left[G_{\kappa}\right]$ are closed under $\kappa$-sequences from $V\left[G_{\kappa}\right]$ and compute the true $\kappa^{++}$, so in the obvious notation

$$
Q_{\kappa}=\left(Q_{\kappa}\right)_{\bar{N}}=\left(Q_{\kappa}\right)_{M} .
$$

We also have

$$
\left(Q_{\kappa}\right)_{N}=(\operatorname{Add}(\kappa, \lambda))_{V\left[G_{\kappa}\right]} .
$$

Letting $g$ factor as $g_{1} \times g_{2}$, where $g_{1}$ is $\left(Q_{\kappa}\right)_{N}$-generic, we build further extensions

$$
\begin{gathered}
k: N\left[G_{\kappa}\right]\left[g_{1}\right] \rightarrow M\left[G_{\kappa}\right][g], \\
\bar{i}: N\left[G_{\kappa}\right]\left[g_{1}\right] \rightarrow \bar{N}\left[G_{\kappa}\right][g], \\
\bar{k}: \bar{N}\left[G_{\kappa}\right][g] \rightarrow M\left[G_{\kappa}\right][g],
\end{gathered}
$$

still preserving the relation $k=\bar{k} \circ \bar{i}$.

The same argument as in stage one allows us to build $H \in V\left[G_{\kappa}\right]\left[g_{1}\right]$ which is $R$-generic over $N\left[G_{\kappa}\right]\left[g_{1}\right]$, where $R$ is the iteration between $\kappa+1$ and $i(\kappa)$ as computed by $N\left[G_{\kappa}\right]$ : We transfer $H$ along $\bar{i}, k$ to get

$$
\begin{aligned}
i: V\left[G_{\kappa}\right] & \rightarrow N\left[i\left(G_{\kappa}\right)\right], & k: N\left[i\left(G_{\kappa}\right)\right] & \rightarrow M\left[j\left(G_{\kappa}\right)\right], \\
\bar{i}: N\left[i\left(G_{\kappa}\right)\right] & \rightarrow \bar{N}\left[\bar{i} \circ i\left(G_{\kappa}\right)\right], & \bar{k}: \bar{N}\left[\bar{i} \circ i\left(G_{\kappa}\right)\right] & \rightarrow M\left[j\left(G_{\kappa}\right)\right],
\end{aligned}
$$

where all the maps are defined in $V\left[G_{\kappa}\right][g]$. Let $l==_{\text {def }} \bar{i} \circ i$.

$\left|P_{\kappa}\right|=\kappa$ and $P_{\kappa}$ is $\kappa$-c.c., so by Fact $2, \S 1.2 .6$, the term forcing

$$
\left(\operatorname{Add}\left(\kappa, \kappa^{++}\right) \times \operatorname{Coll}\left(\kappa, \kappa^{+}\right)\right)_{V^{P_{\kappa}}} / P_{\kappa}
$$

is isomorphic in $V$ to $P$. Applying $i$, we use the $i(P)$-generic $\bar{g}$ to define a generic $g_{a} \times g_{c}^{1}$ for the forcing

$$
\left(\operatorname{Add}\left(i(\kappa), i\left(\kappa^{++}\right)\right) \times \operatorname{Coll}\left(i(\kappa), i\left(\kappa^{+}\right)\right)\right)_{N\left[i\left(G_{\kappa}\right)\right]}
$$

over the model $N\left[i\left(G_{\kappa}\right)\right]$. Using the fact that

$$
V\left[G_{\kappa}\right]\left[g_{1}\right] \vDash{ }^{\kappa} N\left[i\left(G_{\kappa}\right)\right] \subset N\left[i\left(G_{\kappa}\right)\right]
$$

we also build $g_{c}^{0}$ generic over $N\left[i\left(G_{\kappa}\right)\right]$ for the forcing

$$
\left(\operatorname{Coll}\left(\kappa^{+4},<i(\kappa)\right)\right)_{N\left[i\left(G_{\kappa}\right)\right]}
$$

and observe that automatically $g_{c}^{0}$ is mutually generic with $g_{a} \times g_{c}^{1}$.

We transfer $g_{c}^{0}, g_{c}^{1}, g_{a}$ along $\bar{i}$ to get new generics $\bar{g}_{c}^{0}, \bar{g}_{c}^{1}, \bar{g}_{a}$. We need to alter the generic $\bar{g}_{a}$ to get a generic $h_{a}$ such that

$$
\forall p \in g l(p) \in h_{a} .
$$

Recall that conditions in $Q_{\kappa}$ are partial functions $p: \kappa^{++} \times \kappa \rightsquigarrow 2$ with $|\operatorname{dom}(p)|<\kappa$. Therefore $l(p)=l " p$, and so if $q \in h_{a}$ we need to alter its values on the set $l " \kappa^{++} \times \kappa$.

Claim 1. If $q \in l\left(Q_{\kappa}\right)$ then $\left|\operatorname{dom}(q) \cap\left(l “ \kappa^{++} \times \kappa\right)\right| \leq \kappa$ in the model $V\left[G_{\kappa}\right][g]$. Proof. Let $q=l(f)(a)$, where

$$
f:[\kappa]^{|a|} \rightarrow Q_{\kappa} \wedge f \in V\left[G_{\kappa}\right] \wedge a \in\left[\kappa^{++}\right]^{<\omega} .
$$


Define

$$
X_{x}=\left\{\beta<\kappa^{++} \mid \exists \gamma<\kappa(\beta, \gamma) \in \operatorname{dom}(f(x))\right\}
$$

and let $X=\bigcup_{x \in[\kappa]^{|a|}} X_{x}$. Clearly $|X| \leq \kappa$ and

$$
\left\{\beta<\kappa^{++} \mid \exists \gamma<\kappa(i(\beta), \gamma) \in \operatorname{dom}(q)\right\} \subseteq X .
$$

Since $V\left[G_{\kappa}\right][g] \vDash{ }^{\kappa} \bar{N}\left[l\left(G_{\kappa}\right)\right] \subset \bar{N}\left[l\left(G_{\kappa}\right)\right]$, we can alter each condition without getting out of the model $\bar{N}\left[l\left(G_{\kappa}\right)\right]$, and the set of altered conditions $h_{a}$ is in $V\left[G_{\kappa}\right][g]$. It remains to be shown that $h_{a}$ is still $l\left(Q_{\kappa}\right)$-generic. Let $D \in \bar{N}\left[l\left(G_{\kappa}\right)\right]$ be a dense open subset of $l\left(Q_{\kappa}\right)$.

Claim 2. Define an equivalence relation on $l\left(Q_{\kappa}\right)$ by

$$
p \backsim q \Leftrightarrow \operatorname{dom}(p)=\operatorname{dom}(q) \wedge|\{z \mid p(z) \neq q(z)\}| \leq \kappa .
$$

Let

$$
E=\left\{q \in l\left(Q_{\kappa}\right) \mid \forall p \in l\left(Q_{\kappa}\right) p \backsim q \Rightarrow p \in D\right\} .
$$

Then $E$ is dense in $l\left(Q_{\kappa}\right)$.

Proof. Work in $\bar{N}\left[l\left(G_{\kappa}\right)\right]$.We first prove the following fact.

Subclaim 1. If $r \in l\left(Q_{\kappa}\right)$ then there exists $s \leq r$ such that

$$
\forall p \quad p \backsim r \Rightarrow p \cup(s \backslash r) \in D .
$$

Proof. Enumerate $\{X \subseteq \operatorname{dom}(r)|| X \mid \leq \kappa\}$ as $\left\langle X_{\alpha}: \alpha<\mu\right\rangle$, where $\mu<l(\kappa)$. Define by induction a decreasing sequence of conditions $\left\langle r_{\alpha}: \alpha<\mu\right\rangle . r_{0}=r$, and $r_{\beta+1}$ is defined as follows; let $q$ be given by

$$
q(z)= \begin{cases}r_{\beta}(z), & z \in \operatorname{dom}\left(r_{\beta}\right) \backslash X_{\beta}, \\ 1 \backslash r_{\beta}(z), & z \in X_{\beta},\end{cases}
$$

let $\bar{q} \leq q$ be in $D$, and let $r_{\beta+1}=r_{\beta} \cup(\bar{q} \backslash q)$. The forcing $l\left(Q_{\kappa}\right)$ is $l(\kappa)$-closed so define $s$ as a lower bound for $\left\langle r_{\alpha}: \alpha<\mu\right\rangle$. s clearly works.

To finish the proof of the claim start with $r \in l\left(Q_{\kappa}\right)$ and apply the subclaim $\kappa^{+}$times to produce a decreasing sequence $\left\langle s_{\alpha}: \alpha<\kappa^{+}\right\rangle$such that if $p \backsim s_{\alpha}$ then $p \cup\left(s_{\alpha+1} \backslash s_{\alpha}\right) \in D$. Let $q$ be a lower bound for $\left\langle s_{\alpha}: \alpha<\kappa^{+}\right\rangle$. We claim this works. If $\operatorname{dom}(p)=\operatorname{dom}(q)$ and $X=\{z \mid p(z) \neq q(z)\}$ has cardinality $\leq \kappa$ then $X \subseteq \operatorname{dom}\left(s_{\alpha}\right)$ for some $\alpha$. So $p \in D$.

This suffices to show that $h_{a}$ is generic, and a small variation on the argument shows that it is mutually generic with $\bar{g}_{c}^{0} \times \bar{g}_{c}^{1}$.

We now transfer $h_{a}$ along $\bar{k}$ to get $H_{a} \quad j\left(Q_{\kappa}\right)$-generic over $M .(\dagger)$ implies that $\forall p \in g j(p) \in H_{a}$, so we can build maps

$$
\begin{gathered}
j: V\left[G_{\kappa}\right][g] \rightarrow M\left[j\left(G_{\kappa} * g\right)\right], \\
\bar{k}: \bar{N}\left[l\left(G_{\kappa} * g\right)\right] \rightarrow M\left[j\left(G_{\kappa} * g\right)\right], \\
l: V\left[G_{\kappa}\right][g] \rightarrow \bar{N}\left[l\left(G_{\kappa} * g\right)\right],
\end{gathered}
$$

where $j=\bar{k} \circ l$. Define

$$
V^{*}=V\left[G_{\kappa}\right][g], \quad M^{*}=M\left[j\left(G_{\kappa} * g\right)\right], \quad N^{*}=\bar{N}\left[l\left(G_{\kappa} * g\right)\right] .
$$


We argue that $l: V^{*} \rightarrow N^{*}$ is just the ultrapower approximating $j: V^{*} \rightarrow M^{*}$. To do this factor $l$ through $l^{\dagger}: V \rightarrow N^{\dagger}$ the ultrapower approximating $l$, say $l=k^{\dagger} \circ l^{\dagger} . \mathscr{P}_{V^{*}}(\kappa) \subset N^{\dagger}$ and $N^{\dagger} \vDash 2^{\kappa}=\kappa^{++}$so $\kappa_{N^{\dagger}}^{++}=\kappa^{++}$and $\operatorname{crit}\left(k^{\dagger}\right)>\kappa^{++}$. Since $\kappa^{++} \subset \operatorname{rge}\left(k^{\dagger}\right)$ and $N^{*}$ is generated by a $\left(\kappa, \kappa^{++}\right)$extender, $N^{\dagger}=N^{*}$ and we are done.

It remains to be argued that $\bar{g}_{c}^{0} \times \bar{g}_{c}^{1}$ is generic for an appropriate collapse ordering, but this is immediate by the closure of $l\left(Q_{\kappa}\right)$.

So we have produced a model of the hypotheses

(1) $\kappa$ is $\mathscr{P}_{2} \kappa$-hypermeasürable, as witnessed by $j: V \rightarrow M$ with ${ }^{\kappa} M \subset M$, $V_{\kappa+2} \subset M, \operatorname{crit}(j)=\kappa$.

(2) For each strong inaccessible $\alpha \leq \kappa, 2^{\alpha}=\alpha^{++}$and, for all $n$, $0<n<\omega \Rightarrow 2^{\alpha^{+n}}=\alpha^{+n+1}$.

(3) $M \vDash 2^{\kappa}=\kappa^{++} \wedge \forall n \quad 0<n<\omega \Rightarrow 2^{\kappa^{+n}}=\kappa^{+n+1}$.

(4) There is an $F \in V$ which is $P$-generic over $N$, where

$$
P=\operatorname{Coll}\left(\kappa^{+4},<i(\kappa)\right) \times \operatorname{Coll}\left(i(\kappa), i\left(\kappa^{+}\right)\right)_{N}
$$

and $i: V \rightarrow N$ is the ultrapower of $V$ by the ultrafilter

$$
U=\{X \subset \kappa \mid \kappa \in j(X)\} .
$$

(5) $j$ is generated by a $\left(\kappa, \kappa^{+3}\right)$-extender.

We make some incidental remarks

(1) $F \in M$, as it can be coded by an element of $V_{\kappa+2}$.

(2) $\kappa_{N}^{++}=\kappa^{++}$and $\kappa_{M}^{+3}=\kappa^{+3}$.

(3) $\operatorname{crit}(\bar{k})=\kappa_{N}^{+3}<\kappa^{+3}$.

(4) $F$ can be transferred along $\bar{k}$ because it is generic for sufficiently closed forcing.

\section{THE RADIN FORCING}

3.1. Measure sequences. We use the results of the last section to define measure sequences, which will be the building blocks for the modified Radin forcing.

Definition. If $\alpha$ and $\beta$ are inaccessibles,

$$
P(\alpha, \beta)=_{\mathrm{def}} \operatorname{Coll}\left(\alpha^{+4},<\beta\right) \times \operatorname{Coll}\left(\beta, \beta^{+}\right) .
$$

Retaining the notation used in the last paragraph of the preceding section, let $i_{12}: N \rightarrow i(N)$ be $i(i)$, and let $i_{02}: V \rightarrow i(N)$ be $i(i) \circ i$. Since $N \vDash^{i(\kappa)} i(N) \subset$ $i(N)$,

$$
P=i(P)(\kappa, i(\kappa))=i_{02}(P)(\kappa, i(\kappa))
$$

and so we can sensibly define

$$
\begin{aligned}
& P^{* *}=\left\{f:[\kappa]^{2} \rightarrow V_{\kappa} \mid \operatorname{dom}(f) \in U \times U \wedge \forall \alpha, \beta f(\alpha, \beta) \in P(\alpha, \beta)\right\}, \\
& F^{* *}=\left\{f \in P^{* *} \mid i_{02}(f)(\kappa, i(\kappa)) \in F\right\} .
\end{aligned}
$$

Notice that $U$ can be read off from $F^{* *}$ as

$$
U=\left\{X \subseteq \kappa \mid \exists f \in F^{* *} \operatorname{dom}(f)=X \times X\right\} .
$$


We use $j$ and $F^{* *}$ to define our prototypical measure sequence $u$ :

$$
\begin{aligned}
& u(0)=_{\text {def }} \operatorname{crit}(j)=\kappa, \quad u(1)=_{\text {def }} F^{* *}, \\
& u(\beta)=_{\text {def }}\left\{X \in V_{\kappa+1}\right\} u\lceil\beta \in j(X) \quad(\beta>1),
\end{aligned}
$$

where the construction continues for as long as $u \mid \beta \in M$. If the reader is familiar with Radin forcing she will notice that $u$ has in second place the object $F^{* *}$, where putting

$$
\bar{U}=_{\operatorname{def}}\left\{X \subset V_{\kappa} \mid\{\alpha \mid\langle\alpha\rangle \in X\} \in U\right\}
$$

would give a standard Radin sequence as in [R].

The idea behind the definitions that follow is that we use the strong reflection properties of the cardinal $\kappa$ to show that $V_{\kappa}$ contains many objects which resemble $u$. In fact each measure on the sequence $u$ should concentrate on the set of $u$-like sequences.

Definition. $(k, g)$ is a constructing pair iff

(1) $k: V \rightarrow k(V)$ is an elementary embedding into a transitive inner model, and if $\alpha$ is the critical point then ${ }^{\alpha} k(V) \subset k(V)$.

(2) $g$ is $k_{0}(P)\left(\alpha, k_{0}(\alpha)\right)$-generic over $k_{0}(V)$, where $k_{0}$ is the ultrapower approximating $k$ (as usual we factor $k$ through $k_{0}$, say $k=l \circ k_{0}$ ).

(3) $g \in k(V)$.

(4) $g$ can be transferred along $l$ to give a $k(P)(\alpha, k(\alpha))$-generic over $k(V)$.

Definition. If $(k, g)$ is a constructing pair as above then $g^{* *}$ is defined from $g$ in the same way as $F^{* *}$ was defined from $F$, that is

$$
g^{* *}=_{\text {def }}\left\{f \mid k_{02}(f)\left(\alpha, k_{0}(\alpha)\right) \in g\right\},
$$

where $k_{02}=k_{0}\left(k_{0}\right) \circ k_{0}$.

Definition. If $(k, g)$ is a constructing pair as above, then a sequence $w$ is constructed by $(k, g)$ iff

(1) $w \in k(V)$.

(2) $w(0)=\alpha=\operatorname{crit}(k)$.

(3) $w(1)=g^{* *}$.

(4) $w(\beta)=\left\{X \in V_{\alpha+1}|w| \beta \in k(X)\right\} \quad(1<\beta<\operatorname{lh}(w))$.

(5) $k(V) \vDash|\ln (w)| \leq w(0)^{++}$.

$(j, F)$ is the archetypal constructing pair, and for every $\beta<\kappa^{+3}$ it constructs $w\lceil\beta$.

Definition. If $w$ is constructed by some $(k, g)$ then define $\kappa_{w}=w(0)$.

If $\operatorname{lh}(w) \geq 2$ define also

$$
\begin{aligned}
& g_{w}^{* *}==_{\text {def }} w(1), \\
& \mu_{w}={ }_{\operatorname{def}}\left\{X \subset \kappa_{w} \mid \exists f \in g_{w}^{* *} \operatorname{dom}(f)=[X]^{2}\right\}, \\
& \bar{\mu}_{w}={ }_{\operatorname{def}}\left\{X \subset V_{\kappa_{w}} \mid\{\alpha \mid\langle\alpha\rangle \in X\} \in \mu_{w}\right\}, \\
& g_{w}={ }_{\operatorname{def}}\left\{[f]_{\mu_{w} \times \mu_{w}} \mid f \in g_{w}^{* *}\right\}, \\
& \mathscr{F}_{w}==_{\operatorname{def}} \bar{\mu}_{w} \cap \bigcap\{w(\alpha) \mid 1<\alpha<\operatorname{lh}(w)\},
\end{aligned}
$$


where $[f]_{\mu_{w} \times \mu_{w}}$ is the set represented by the function $f$ in the ultrapower $V^{\kappa_{w} \times \kappa_{w}} / \mu_{w} \times \mu_{w}$.

Of course, given a particular $(k, g)$ which constructs $w$ then $g_{w}=g$ and so forth, but the point is that these definitions are independent of the choice of a constructing pair for $w$.

Definition. Define inductively classes of sequences

$$
\begin{aligned}
U_{0} & =\operatorname{def}\{w \mid \exists(k, g) \quad(k, g) \text { constructs } w\}, \\
U_{n+1} & =\operatorname{def}\left\{w \in U_{n} \mid U_{n} \cap V_{\kappa_{w}} \in \mathscr{F}_{w}\right\}, \quad U_{\infty}=\operatorname{def} \bigcap_{n \in \omega} U_{n} .
\end{aligned}
$$

Lemma 1. For every $\alpha<\kappa^{+3}, u \uparrow \alpha$ exists and is a member of $U_{\infty}$.

Proof. It is routine to check that if $\alpha<\kappa^{+3}$ then $u \uparrow \alpha$ can be coded by an element of $V_{\kappa+2}$, and hence $u \uparrow \alpha \in M$ and $u(\alpha)$ exists.

Before proving the second half we unravel the definition of $U_{\infty}$.

Claim 1. $\forall \alpha<\kappa^{+3} u \uparrow \alpha \in U_{\infty}$ if and only if $\forall \alpha<\kappa^{+3} \forall n<\omega M_{n} \vDash u \uparrow \alpha \epsilon$ $U_{0}$, where $M_{n}$ is the $n$th iterate of $V$ by $j$.

Proof. This is quite easy by induction.

$$
\begin{aligned}
\forall \alpha< & \kappa^{+3} \quad u\left\lceil\alpha \in U_{n+1}\right. \\
& \Leftrightarrow \forall \alpha<\kappa^{+3} \quad U_{n} \cap V_{\kappa} \in u(\alpha) \\
& \Leftrightarrow \forall \alpha<\kappa^{+3} \quad u\left\lceil\alpha \in j\left(U_{n}\right)\right. \\
& \Leftrightarrow \forall \alpha<\kappa^{+3} \quad M \vDash u\left\lceil\alpha \in U_{n}\right. \\
& \Leftrightarrow \forall \alpha<\kappa^{+3} \quad M_{n+1} \vDash u\left\lceil\alpha \in U_{0} .\right.
\end{aligned}
$$

Fix $\alpha<\kappa^{+3}$. We prove that $M \vDash u\left\lceil\alpha \in U_{0}\right.$, and then use the agreement between $M$ and $M_{n}$ for $n>1$ to argue that this is enough.

Fix $\beta$ such that, if $j_{\beta}: V \rightarrow M_{\beta}$ is the elementary embedding obtained by restricting the extender $E$ which describes $j$ to $E_{\beta}=E \uparrow[\beta]^{<\omega}$, then

(1) $\kappa^{++}<\beta<\kappa^{+3}$ and $\beta>\alpha$.

(2) ${ }^{\kappa} M_{\beta} \subset M_{\beta}$.

(3) $u\left\lceil\alpha\right.$ and $F$ are in $M_{\beta}$.

By the choice of $\beta, \kappa_{M_{\beta}}^{++}=\kappa^{++}$and $\alpha<\kappa_{M_{\beta}}^{+++}$. Also $E_{\beta} \in M$, and if $j_{\beta}^{*}: M \rightarrow j_{\beta}^{*}(M)$ is the embedding which $M$ computes from $E_{\beta}$ then $j_{\beta}^{*}=j_{\beta}\left\lceil M\right.$ and the models $M_{\beta}, j_{\beta}^{*}(M)$ agree up to rank $\beta$.

Using this information it is routine to check that

$$
M \vDash "\left(j_{\beta}^{*}, F\right) \text { constructs } u \uparrow \alpha . "
$$

To finish the proof observe that if $j_{1 n}: M \rightarrow M_{n}$ is the map arising from the iteration of $j$ then

$$
\operatorname{crit}\left(j_{1 n}\right)=j(\kappa)>\kappa^{+3}>\operatorname{rank}(u\lceil\alpha),
$$

so $M_{n} \vDash u \uparrow \alpha \in U_{0}$. 
Because the measures on a measure sequence are countably complete, if $w \in U_{\infty}$ and $\operatorname{lh}(w) \geq 2$ then $U_{\infty} \cap V_{\kappa} \in \mathscr{F}_{w}$.

3.2. The forcing poset. Given $w \in U_{\infty}$ with $\ln (w) \geq 2$ we define a poset $Q_{w}$. The forcing which we eventually use to establish the theorem will be $Q_{u \uparrow \beta}$ for a well-chosen value of $\beta$, but it is advantageous to do the proofs uniformly for all $Q_{w}$.

Definition. $p$ is a condition in $Q_{w}$ iff $p=\left\langle X_{i}: i<n\right\rangle$, where $n>1$ and

(1) $X_{0}=\left\langle\kappa_{0}\right\rangle$ for some $\kappa_{0}<\kappa$.

(2) For $0<i<n-1, X_{i}$ is

- EITHER a pair $\left(P_{i}, \kappa_{i}\right)$, where

$$
\kappa_{i}<\kappa, \quad P_{i} \in P\left(\kappa_{i-1}, \kappa_{i}\right),
$$

- OR a quadruple $\left(w_{i}, A_{i}, H_{i}, h_{i}\right)$, where

$w_{i} \in U_{\infty}$ and $\operatorname{lh}\left(w_{i}\right)>1, \quad \kappa_{i}=_{\operatorname{def}} \kappa_{w_{i}}$,

$A_{i} \in \mathscr{F}_{w_{i}}, \quad H_{i} \in g_{w_{i}}^{* *}$,

$h_{i}$ is a function, $\quad \operatorname{dom}\left(h_{i}\right)=\left\{\kappa_{v} \mid v \in A_{i}\right\} \backslash\left(\kappa_{i-1}+1\right)$,

$\forall \alpha \in \operatorname{dom}\left(h_{i}\right) \quad h_{i}(\alpha) \in P\left(\kappa_{i-1}, \alpha\right), \quad \operatorname{dom}\left(H_{i}\right)=\left[\operatorname{dom}\left(h_{i}\right)\right]^{2}$.

(3) $\kappa_{0}<\kappa_{1}<\cdots<\kappa_{n-1}<\kappa$.

(4) $X_{n}$ is a quadruple $\left(w_{n}, A_{n}, H_{n}, h_{n}\right)$ with the same properties as above, such that $w_{n}=w$.

Before giving the formal definition of the ordering on the conditions we give some informal motivation. The intended generic object is a pair of sequences $(\vec{v}, \vec{F})$, where

(1) $\ln (\vec{v})=\ln (\vec{F})$.

(2) $\forall \alpha<\operatorname{lh}(\vec{v}) v(\alpha) \in U_{\infty}$. (Let $\left.\kappa_{\alpha}=\kappa_{v(\alpha)} \cdot\right)$

(3) $\alpha \mapsto \kappa_{\alpha}$ is monotone increasing, and $C=\left\{\kappa_{\alpha} \mid \alpha<\ln (\vec{v})\right\}$ is closed unbounded in $\kappa_{w}$.

(4) For $\alpha<\operatorname{lh}(\vec{v}), F(\alpha)$ is a $P\left(\kappa_{\alpha}, \kappa_{\alpha+1}\right)$-generic filter.

The typical condition $p=\left\langle X_{i}: i<n\right\rangle$ of the last definition is intended to put the following constraints on $(\vec{v}, \vec{F})$ :

(1) $v_{0}=\kappa_{0}$.

(2) If $0<i<n-1$ and $X_{i}$ is a pair $\left(P_{i}, \kappa_{i}\right)$ then, for some $\alpha<\operatorname{lh}(\vec{v})$, $\kappa_{i-1}=\kappa_{\alpha}, \kappa_{i}=\kappa_{\alpha+1}$, and $P_{i} \in F(\alpha)$.

(3) If $0<i<n$ and $X_{i}$ is a quadruple $\left(w_{i}, A_{i}, H_{i}, h_{i}\right)$ then $\kappa_{i}$ is a limit point $\kappa_{\lambda}$ in $C$. Let $\kappa_{i-1}=\kappa_{\alpha}$. Then $h_{i}\left(\kappa_{\alpha+1}\right) \in F(\alpha)$, and if $\alpha<\beta<\lambda$ we have $v(\beta) \in A_{i}$ and $H\left(\kappa_{\beta}, \kappa_{\beta+1}\right) \in F(\beta)$.

Definition. Let $X$ be a tuple.

(1) $u_{X}=\langle\delta\rangle$ if $X=\langle\delta\rangle$ or $X=\langle P, \delta\rangle$.

(2) $u_{X}=w$ if $X=\langle w, A, H, h\rangle$.

(3) $\kappa_{X}=\kappa_{u_{X}}$. 
Definition. Let $p=\left\langle X_{i}: i<m\right\rangle$ and $q=\left\langle Y_{j}: j<n\right\rangle$. $p$ extends $q$ iff

(1) $m \geq n$.

(2) $\left\{\kappa_{X_{0}}, \ldots, \kappa_{X_{m-1}}\right\} \supseteq\left\{\kappa_{Y_{0}}, \ldots, \kappa_{Y_{n-1}}\right\}$.

(3) $\kappa_{X_{0}}=\kappa_{Y_{0}}, X_{0}=Y_{0}$.

(4) For $0<i<m$, if $X_{i}$ is a pair $\langle P, \alpha\rangle$ then

- EITHER there is $j<n$ such that $Y_{j}=\langle Q, \alpha\rangle$, and $P \leq Q$ (this will make sense because other clauses of the definition will ensure that in this case $\kappa_{X_{i-1}}=\kappa_{Y_{j-1}}$, so $P$ and $Q$ are in the same partial ordering),

- OR there is no $j$ with $\alpha=\kappa_{Y_{j}}$ and for the least $j$ such that $\alpha<\kappa_{Y_{j}}$

$$
\begin{aligned}
& Y_{j} \text { is a quadruple }\langle u, A, H, h\rangle, \\
& \alpha \in A, \\
& \text { IF } \kappa_{X_{i-1}}=\kappa_{Y_{j-1}} P \leq h(\alpha), \\
& \text { IF } \kappa_{X_{i-1}}>\kappa_{Y_{j-1}} P \leq H\left(\kappa_{X_{i-1}}, \alpha\right) .
\end{aligned}
$$

(5) For $0<i<m$, if $X_{i}$ is a quadruple $\langle u, A, H, h\rangle$ then

- EITHER there is $j<n$ such that $Y_{j}=\langle u, B, I, i\rangle$, in which case

$$
\begin{aligned}
& A \subseteq B, \\
& \forall(\alpha, \beta) \in \operatorname{dom}(h) \quad H(\alpha, \beta) \leq I(\alpha, \beta)
\end{aligned}
$$

and

$$
\begin{aligned}
& \text { IF } \kappa_{X_{i-1}}=\kappa_{Y_{j-1}} \forall \alpha \in \operatorname{dom}(h) h(\alpha) \leq i(\alpha), \\
& \text { IF } \kappa_{X_{i-1}}>\kappa_{Y_{j-1}} \forall \alpha \in \operatorname{dom}(h) h(\alpha) \leq I\left(\kappa_{X_{i-1}}, \alpha\right),
\end{aligned}
$$

- OR there is no $j$ with $\kappa_{X_{i}}=\kappa_{Y_{j}}$, and for the least $j$ such that $\kappa_{X_{i}}<\kappa_{Y_{j}}$

$Y_{j}$ is a quadruple $\langle v, C, J, j\rangle$,

$u \in C, \quad A \subseteq C$,

$\forall(\alpha, \beta) \in \operatorname{dom}(H) \quad H(\alpha, \beta) \leq J(\alpha, \beta)$,

IF $\kappa_{X_{i-1}}=\kappa_{Y_{j-1}} \forall \alpha \in \operatorname{dom}(h) h(\alpha) \leq j(\alpha)$,

IF $\kappa_{X_{i-1}}>\kappa_{Y_{j-1}} \forall \alpha \in \operatorname{dom}(h) h(\alpha) \leq J\left(\kappa_{X-1}, \alpha\right)$.

Remark. $Q_{w}$ has $\kappa_{w}^{++}$-c.c.

Proof. Let $\left\langle p_{\alpha}: \alpha\left\langle\kappa_{w}^{++}\right\rangle\right.$be an antichain in $Q_{w}$, and let us write

$$
p_{\alpha}=x_{\alpha} \frown\left\langle w, A_{\alpha}, H_{\alpha}, h_{\alpha}\right\rangle .
$$

Since $x_{\alpha} \in V_{\kappa_{w}}$, we may assume that $x_{\alpha}$ is some fixed sequence $x$. Incompatibility can only arise from disagreement between the $h_{\alpha}$, and $p_{\alpha} \perp p_{\beta}$ implies $h_{\alpha}$ and $h_{\beta}$ represent incompatible conditions in the ultrapower by $\mu_{w}$. Since they represent conditions in a forcing notion of size $\kappa_{w}^{++}$we have a contradiction and the claim follows. 


\subsection{The generic object.}

Definition. A sequence $u \in U_{\infty}$ occurs in a condition $p=\left\langle X_{i}: i<m\right\rangle$ iff there is $i<m-1$ such that one of the following holds:

(1) $i=0$ and $u=X_{0}$.

(2) $X_{i}=\langle P, \delta\rangle$ and $u=\langle\delta\rangle$.

(3) $X_{i}=\langle u, A, H, h\rangle$.

Definition. $P$ occurs in $p=\left\langle X_{i}: i<m\right\rangle$ iff there is $i$ with $0<i<m$ such that $X_{i}=\langle P, \delta\rangle$ for some $\delta$.

Notice that $w$ does not occur in the conditions of $Q_{w}$.

Definition. Let $G$ be a $Q_{w}$-generic filter. Define a pair of sequences $(\langle v(\alpha): \alpha<$ $\lambda\rangle,\langle F(\alpha): \alpha<\lambda\rangle)$

(1) $\langle v(\alpha): \alpha<\lambda\rangle$ enumerates $\left\{v \in U_{\infty} \mid \exists p \in G \quad v\right.$ occurs in $\left.p\right\}$ so that $\alpha \mapsto \kappa_{\alpha}=\kappa_{v(\alpha)}$ is increasing.

(2) $F(\alpha)=\left\{P \in P\left(\kappa_{\alpha}, \kappa_{\alpha+1}\right) \mid \exists p \in G \quad P\right.$ occurs in $\left.p\right\}$.

It is routine to check that the generic filter $G$ and the pair of sequences $(\vec{v}, \vec{F})$ are each definable from the other.

We briefly develop the basic properties of the forcing; the proofs are very similar to those for Radin forcing (see [R]).

Lemma 1. Let $G$ be $Q_{w}$-generic and let $(\vec{v}, \vec{F})$ and $\vec{\kappa}$ be defined as above. Then $C=\left\{\kappa_{\alpha} \mid \alpha<\ln (\vec{v})\right\}$ is a closed unbounded subset of $\kappa_{w}$.

Proof. First, we prove that $C$ is closed. Let $p \in Q_{w}$ be a condition and $\alpha<\kappa_{w}$ an ordinal such that $p \Vdash \alpha \notin C$. No sequence $v$ with $\kappa_{v}=\alpha$ occurs in $p$, so consider $X$ the first entry of $p$ with $\kappa_{X}>\alpha$. If $X$ is a pair then $p$ forces $\alpha$ is not a limit point of $C$. If $X$ is a quadruple $\langle u, A, H, h\rangle$ then define $p^{\prime} \leq p$ by shrinking $A$ to $A^{\prime}=A \backslash V_{\alpha}$, so that $p^{\prime}$ forces $\alpha$ is not a limit point of $C$.

To prove that $C$ is unbounded, let $\beta<\kappa_{w}$, and $p \in Q_{w}$, say $p=x \frown$ $\langle w, A, H, h\rangle$. Since $A \in \bar{\mu}_{w}$, there is $\langle\alpha\rangle \in A$ with $\alpha>\beta$, so $p$ can be extended to

$$
p^{\prime}=x \frown\langle h(\alpha), \alpha\rangle \frown\langle w, A, H, H(\alpha)\rangle
$$

and $p^{\prime}$ forces that $\beta$ is not a bound for $C$.

The lemma is proved.

We make the remark that if $\operatorname{lh}(w) \geq 3$ then we could also have proved the "unbounded" clause by showing that many quadruples could be added to the condition $p$. (See the next lemma.)

Lemma 2. With the same assumptions as in Lemma 1, we assert the following:

(1) If $\mu<\lambda$ is such that $\ln (v(\mu))>1$ then the pair $(\vec{v}|\mu, \vec{F}| \mu)$ is generic for the forcing $Q_{v(\mu)}$.

(2) $\forall A \in V_{\kappa_{w}+1} \quad\left(A \in \mathscr{F}_{w} \Leftrightarrow \exists \beta \quad \forall \alpha>\beta \quad v(\alpha) \in A\right)$.

(3) $\forall H \in g_{w}^{* *} \exists \beta \quad \forall \alpha>\beta \quad H\left(\kappa_{\alpha}, \kappa_{\alpha+1}\right) \in F(\alpha)$.

Proof. (1) follows immediately from the observation that if $p$ is a condition in which $u_{\mu}$ appears then $Q_{w}\lceil p$ factors into two parts, of which the first is $Q_{u_{\mu}} \backslash p^{\prime}$ for $p^{\prime} \in Q_{u_{\mu}}$ an initial segment of $p$. 
For (2), first assume that $B \in \mathscr{F}_{w}$. Let $p=x \frown\langle w, A, H, h\rangle$ be some condition; then the condition

$$
p^{\prime}=x \frown\langle w, A \cap B, H, h\rangle
$$

is an extension of $p$ forcing that a final segment of $\vec{v}$ lies in $B$. For the other direction, assume towards a contradiction that $B$ contains a final segment of $\vec{v}$ but $B \notin \mathscr{F}_{w}$. The "unbounded" clause of the last lemma shows that $V_{\kappa} \backslash B \notin \bar{\mu}_{w}$, so we must have $V_{\kappa} \backslash B \in w(\beta)$ for $\beta>1$.

Subclaim 1. If $\beta>1$ and $B \in w(\beta)$ then $v(\alpha) \in B$ for an unbounded set of $\alpha<\lambda$.

Proof. We show that if $\alpha<\kappa_{w}$ and $q=x \frown\langle w, A, H, h\rangle$ is a condition then there exist the set of $v$ for which $\kappa_{v}>\alpha$ and $B, I, i$ such that $(v, B, I, i)$ can be added to $q$ is a measure one set for $w(\beta)$. This clearly suffices.

Fix $(k, g)$ as a constructing pair for $w$. By definition, it is enough to show that in $k(V)$ there are $B, I, i$ such that $\langle w \mid \beta, B, I, i\rangle$ can be added to

$$
k(q)=x \frown\langle k(w), k(A), k(H), k(h)\rangle .
$$

We claim that $A, H, h$ will work (they exist in $k(V)$ because they are all elements of $\left.V_{\kappa_{w}+1}\right)$.

(1) $A \in w(\beta)$, so $w\lceil\beta \in k(A)$.

(2) $A=i(A) \cap V_{\kappa_{w}} \subset i(A)$.

(3) $H=i(H)\left\lceil\kappa_{w} \times \kappa_{w}\right.$.

(4) $h=i(h)\left\lceil\kappa_{w}\right.$.

The subclaim is proved.

This gives a contradiction, so (2) is proved.

Finally, let $I \in g_{w}^{* *}$. From (2) $\left(\kappa_{\alpha}, \kappa_{\alpha+1}\right) \in \operatorname{dom}(I)$ for large enough $\alpha$, and an argument parallel to that for (a) shows that any condition refines to one which forces $I\left(\kappa_{\alpha}, \kappa_{\alpha+1}\right) \in F_{\alpha}$ on a final segment. The lemma is proved.

3.4. The Prikry property. We prove that questions about the generic extension by some $Q_{w}$ can be decided in a simple way; this will enable us to prove that the forcing does not do too much damage to the universe.

Definition. If $p$ and $q$ are conditions in $Q_{w}$ then $q$ is a direct extension of $p$ iff $q \leq_{Q_{w}} p$ and $\operatorname{lh}(q)=\operatorname{lh}(p)$. We write $q \leq_{d} p$ in this case.

Theorem 1. Let $b \in R O\left(Q_{w}\right)$ be a Boolean value, and let $p \in Q_{w}$ be some condition. Then there is $q \leq_{d} p$ such that $q \| b$.

Proof. The strategy of the proof is to write $p$ as $z \frown\langle w, A, H, h\rangle$, show that $b$ is decided by some extension $z^{\prime} \frown\left\langle w, A^{\prime}, H^{\prime}, h^{\prime}\right\rangle \leq p$ in which no sequences have been added past the top of $z$, and then make an appeal to induction and the uniform definition of the forcing. So fix $p=p^{0}=z^{0} \frown\left\langle w, A^{0}\right.$, $\left.H^{0}, h^{0}\right\rangle$. Also fix $(k, g)$ a constructing pair for $w$.

We factor $k$ through $k_{0}$, the ultrapower by $\mu_{w}$, to get $k=l \circ k_{0}$.

\section{Definition.}

(1) $P_{1}^{*}=\left\{f \in k_{0}(V) \mid \operatorname{dom}(f) \in k_{0}\left(\mu_{w}\right) \wedge \forall \alpha f(\alpha) \in k_{0}(P)\left(\kappa_{w}, \alpha\right)\right\}$.

(2) $g^{*}=\left\{f \in P_{1}^{*} \mid[f]_{k_{0}\left(\mu_{w}\right)} \in g\right\}$. 
(3) $P^{*}=l\left(P_{1}^{*}\right)$.

(4) $P^{* *}=\{F \in V \mid \forall(\alpha, \beta) \in \operatorname{dom}(F) F(\alpha, \beta) \in P(\alpha, \beta)\}$.

Equip $P_{1}^{*}$ with the pre-ordering

$$
f \leq g \Leftrightarrow\{\alpha \mid f(\alpha) \leq g(\alpha)\} \in k_{0}\left(\mu_{w}\right)
$$

and equip $P^{*}$ similarly. By the definition of constructing pair $g^{*}$ is $P_{1}^{*}$-generic over $k_{0}(V)$ and $l^{\prime \prime} g^{*}$ generates a $P^{*}$-generic over $k(V)$.

Definition. $x$ is a lower part iff $x$ is a proper initial segment of some condition in $Q_{w}$.

Definition. If $x$ is a lower part then $\kappa_{x}={ }_{\text {def }} \kappa_{X}$ for $X$ the last tuple in $x$.

Definition. Let $F, G$ be elements of $P^{* *}$.

(1) $F \leq G$ iff $\forall(\alpha, \beta) \in \operatorname{dom}(F) F(\alpha, \beta) \leq G(\alpha, \beta)$. $^{1}$

(2) $F \leq^{*} G$ iff $\{(\alpha, \beta) \mid F(\alpha, \beta) \leq G(\alpha, \beta)\} \in \mu_{w} \times \mu_{w}$.

In the first case we say that $F$ refines $G$, in the second that $F$ refines $G$ almost everywhere.

Similarly, let $f, g$ be functions with domain in $\mu_{w}$ such that for some $\delta<\kappa \quad \forall \alpha \quad f(\alpha), g(\alpha) \in P(\delta, \alpha)$.

(1) $f \leq g$ iff $\forall \alpha \in \operatorname{dom}(f) \quad f(\alpha) \leq g(\alpha)$.

(2) $f \leq^{*} g$ iff $\{\alpha \mid f(\alpha) \leq g(\alpha)\} \in \mu_{w}$.

Step One. We find $A^{1} \subseteq A^{0}, H^{1} \leq H^{0}, h^{1} \leq h^{0}$, such that

$$
p^{1}=z^{0} \frown\left\langle w, A^{1}, H^{1}, h^{1}\right\rangle
$$

has the following property; if $z \frown X \frown\langle w, B, I, i\rangle$ is an extension of $p^{1}$ deciding $b$ and having $\kappa_{X}>\kappa_{z^{0}}$ then there exist $\bar{A} \subset A^{1}, \bar{H} \leq H^{1}$ such that $z \frown X \frown\left\langle w, \bar{A}, \bar{H}, \bar{H}\left(\kappa_{X}\right)\right\rangle$ decides $b$.

Construction. Consider conditions in $k\left(Q_{w}\right)$ of the form

$$
z \frown X \frown\langle k(w), A, H, h\rangle,
$$

where $z$ is a lower part and $X$ is a tuple such that $u_{X}=w \uparrow \alpha$ for some $0<\alpha<\operatorname{lh}(w)$. Notice that $h \in P^{*}$.

Say that $h \in P^{*}$ is good if for all lower parts $z \in V_{\kappa_{w}}$ and all $X$ such that $\exists \alpha<\operatorname{lh}(w) \quad u_{X}=w\lceil\alpha$,

$$
\begin{gathered}
\exists A \quad \exists H \quad \exists h^{\prime} \leq h \quad z \frown X \frown\left\langle k(w), A, H, h^{\prime}\right\rangle \| k(b) \\
\Rightarrow \exists A^{\prime} \exists H^{\prime} z \frown X \frown\left\langle k(w), A^{\prime}, H^{\prime}, h\right\rangle \| k(b) .
\end{gathered}
$$

Claim 1. The set of good $h$ is a dense subset of $P^{*}$ lying in $k(V)$.

Proof of Claim 1. Work in $k(V)$. There are $\kappa_{w}$ possibilities for $z$. From the definition of constructing pair $w \in k(V)$ and

$$
k(V) \vDash|\operatorname{lh}(w)| \leq \kappa_{w}^{++} .
$$

Also for each $\alpha$ there are only $2^{\kappa_{w}}=\kappa_{w}^{++} X$ such that $u_{X}=w \uparrow \alpha$. So enumerate the possible pairs $(z, X)$ as $\left\langle\left(z_{\gamma}, X_{\gamma}\right): \gamma<\kappa_{w}^{++}\right\rangle$. Fix $f \in P^{*}$, and

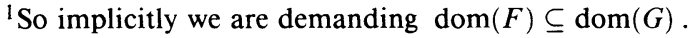


define a descending sequence in $P^{*}$ by setting $f_{0}=f$ and defining $f_{\gamma+1}$ to be some $g \leq f_{\gamma}$ such that

$$
\exists A \exists H \quad z_{\gamma} \frown X_{\gamma} \frown\langle k(w), A, H, g\rangle \| k(b)
$$

if such exists, and $f_{\gamma+1}=f_{\gamma}$ otherwise. Let $f^{\prime} \in P^{*}$ be a lower bound for the sequence $\left\langle p_{\gamma}: \gamma<\kappa_{w}^{++}\right\rangle$. Now let $z, X, A, H, h^{\prime} \leq f^{\prime}$ be such that

$$
z \frown X \frown\left\langle k(w), A, H, h^{\prime}\right\rangle \| k(b) .
$$

$(z, X)$ is enumerated as $\left(z_{\gamma}, X_{\gamma}\right)$ for some $\gamma . f_{\gamma+1}$ was defined, so for some $B, I$

$$
z \frown X \frown\left\langle k(w), B, I, f_{\gamma+1}\right\rangle \| k(b) .
$$

$f^{\prime} \leq f_{\gamma+1}$, so $B, I$ witness that $f^{\prime}$ is good.

Since $g^{*}$ is generic we can find $F \in g^{* *}$ such that $F \leq H^{0}$ and $k(F)\left(\kappa_{w}\right)$ is good. Now the set $R$ of those $u$ such that

$$
\begin{aligned}
\forall z \forall X\left[u_{X}=u \wedge \exists A \exists H \quad \exists h^{\prime} \leq F\left(\kappa_{u}\right) z \frown X \frown\left\langle w, A, H, h^{\prime}\right\rangle \| b\right. \\
\left.\Rightarrow \exists A^{\prime} \exists H^{\prime} z \frown X \frown\left\langle w, A^{\prime}, H^{\prime}, F\left(\kappa_{u}\right)\right\rangle \| b\right]
\end{aligned}
$$

is by construction a member of the filter $\mathscr{F}_{w}$.

Define

$$
\begin{aligned}
A^{1} & =A^{0} \cap R, \\
h^{1} & =h^{0} \uparrow\left\{\kappa_{v} \mid v \in A^{1}\right\} \backslash\left(\kappa_{z^{0}}+1\right), \\
H^{1} & =F\left\lceil\left[\operatorname{dom}\left(h^{1}\right)\right]^{2} .\right.
\end{aligned}
$$

We prove that $p^{1}$ has the right behaviour. If $z \frown X \frown\langle w, B, I, i\rangle$ is an extension of $p^{1}$ deciding $b$ and having $\kappa_{X}>\kappa_{z^{0}}$ then $u_{X}$ is chosen from $R$ and $i \leq F\left(\kappa_{X}\right)$ so we can find $A^{\prime}$ and $H^{\prime}$ as in the definition of $R$. It is easy to alter $A^{\prime}, H^{\prime}$ to $\bar{A}, \bar{H}$ with the right properties.

Step Two. We find $A^{2} \subseteq A^{1}, H^{2} \leq H^{1}, h^{2} \leq h^{1}$, such that

$$
p^{2}=z^{0} \frown\left\langle w, A^{2}, H^{2}, h^{2}\right\rangle
$$

has the following property; if $z \frown X \frown\langle w, B, I, i\rangle$ is an extension of $p^{2}$ deciding $b$ and having $\kappa_{X}>\kappa_{z^{0}}$ then $z \frown X \frown\left\langle w, A^{2}, H^{2}, H^{2}\left(\kappa_{X}\right)\right\rangle$ decides $b$.

Construction. For each $\alpha<\kappa_{w}$ there are fewer than $\kappa_{w}$ lower parts $z$ with $\kappa_{z}=\alpha$. Fix $\alpha$ for the moment, and for each $z$ such that $\kappa_{z}=\alpha$ and

$$
\exists B \subseteq A^{1} \exists G \leq H^{1} z \frown\left\langle w, B, G, H^{1}\left(\kappa_{z}\right)\right\rangle \| b
$$

choose an appropriate pair $\left(B^{z}, G^{z}\right)$. Using genericity find $F_{\alpha} \in g^{* *}$ such that $F_{\alpha} \leq G^{z}$ for all $z$ with $\kappa_{z}=\alpha$. Find $C_{\alpha} \in \mu_{\omega}$ with $\left[C_{\alpha}\right]^{2} \subset \operatorname{dom}\left(F_{\alpha}\right)$.

$$
E_{\alpha}=\operatorname{def} A^{1} \cap \bigcap_{z, \kappa_{z}=\alpha} B^{z} \cap\left\{v \mid\left\langle\kappa_{v}\right\rangle \in C_{\alpha}\right\} .
$$

By the usual argument using the constructing pair $E_{\alpha} \in \mathscr{F}_{w}$. By genericity again find $F \in g^{* *}, F \leq H^{1}$, such that

$$
\forall \alpha<\kappa_{w} \quad F \leq^{*} F_{\alpha} .
$$


Find $D_{\alpha} \in \mu_{w}$ such that

$$
\forall(\gamma, \delta) \in\left[D_{\alpha}\right]^{2} F(\gamma, \delta) \leq F_{\alpha}(\gamma, \delta) .
$$

Let

$$
\begin{aligned}
E_{\alpha}^{\prime} & =E_{\alpha} \cap\left\{v \mid\left\langle\kappa_{v}\right\rangle \in D_{\alpha}\right\}, \\
A^{2} & =\left\{v \in A^{1} \mid \forall \alpha<\kappa_{w} \quad v \in E_{\alpha}^{\prime}\right\}, \\
h^{2} & =h^{1}\left\lceil\left\{\kappa_{v} \mid v \in A^{2}\right\},\right. \\
H^{2} & =F\left\lceil\left[\operatorname{dom}\left(h^{2}\right)\right]^{2},\right. \\
p^{2} & =z^{0} \frown\left\langle w, A^{2}, H^{2}, h^{2}\right\rangle .
\end{aligned}
$$

We claim that $p^{2}$ has the property we demanded. To show this let $z \frown X \frown$ $\langle w, B, I, i\rangle$ be an extension of $p^{2}$ deciding $b$ and having $\kappa_{X}>\kappa_{z^{0}}$. Let $x=z \frown X$. Now by the work we did in Step One and the definitions above

$$
z \frown X \frown\left\langle w, B^{x}, G^{x}, H^{2}\left(\kappa_{X}\right)\right\rangle \| b .
$$

Therefore it will be enough to show that

$$
z \frown X \frown\left\langle w, A^{2}, H^{2}, H^{2}\left(\kappa_{X}\right)\right\rangle \leq z \frown X \frown\left\langle w, B^{x}, G^{x}, H^{2}\left(\kappa_{X}\right)\right\rangle,
$$

which amounts to showing that anything which can be added between $X$ and the top on the LHS can also be added on the RHS.

If $v \in A^{2}$, and $\kappa_{v}>\kappa_{X}=\kappa_{x}$, then $v \in E_{\kappa_{x}}^{\prime}$ and therefore $v \in B^{x}$.

If $v, w \in A^{2}$, and $\kappa_{x}<\kappa_{v}<\kappa_{w}$, then $v, w \in E_{\kappa_{x}}^{\prime}$ and so by definition $\kappa_{v}, \kappa_{w} \in C_{\kappa_{x}} \cap D_{\kappa_{x}}$. Therefore

$$
\begin{aligned}
H^{2}\left(\kappa_{v}, \kappa_{w}\right) & =F\left(\kappa_{v}, \kappa_{w}\right) \\
& \leq F_{\kappa_{x}}\left(\kappa_{v}, \kappa_{w}\right) \quad \text { because }\left(\kappa_{v}, \kappa_{w}\right) \in\left[D_{\kappa_{x}}\right]^{2} \\
& \leq G^{x}\left(\kappa_{v}, \kappa_{w}\right) \quad \text { because }\left(\kappa_{v}, \kappa_{w}\right) \in\left[C_{\kappa_{x}}\right]^{2} .
\end{aligned}
$$

So the constraint imposed by $A^{2}$ and $H^{2}$ is more stringent than that imposed by $B^{x}$ and $G^{x}$, and the claim is proved.

Step Three. We find $A^{3} \subseteq A^{2}, H^{3} \leq H^{2}, h^{3} \leq h^{2}$ such that if

$$
p^{3}=z^{0} \frown\left\langle w, A^{3}, H^{3}, h^{3}\right\rangle
$$

and

$$
z^{\prime} \frown X \frown\langle P, \delta\rangle \frown\left\langle w, A^{\prime}, H^{\prime}, h^{\prime}\right\rangle \leq p^{3}
$$

is a condition deciding $b, \kappa_{z^{0}}<\kappa_{X}<\delta$, then

$$
z^{\prime} \frown X \frown\left\langle H^{3}\left(\kappa_{X}, \delta\right), \delta\right\rangle \frown\left\langle w, A^{3}, H^{3}, H^{3}(\delta)\right\rangle \| b .
$$

Construction. We define embeddings $k^{12}=k(k), k^{02}=k(k) \circ k$. Consider conditions in $k^{02}\left(Q_{w}\right)$ of the form

$$
z \frown X \frown\left\langle P, k\left(\kappa_{w}\right)\right\rangle \frown\left\langle k^{02}(w), k^{02}\left(A^{2}\right), k^{02}\left(H^{2}\right), k^{02}\left(H^{2}\right)\left(k\left(\kappa_{w}\right)\right)\right\rangle,
$$

where $z$ is a lower part and $u_{X}=w\lceil\alpha$ for some $\alpha$ with $0<\alpha<\operatorname{lh}(w)$. $P \in P\left(\kappa_{w}, k\left(\kappa_{w}\right)\right)_{k^{02}(V)}$, and by the definition of constructing pair $l^{\prime \prime} g$ generates a generic over $k(V)$ for this forcing.

An argument exactly parallel to that of Step One enables us to define $A^{3}$, $H^{3}, h^{3}$. 
Step Four. We find $A^{4} \subseteq A^{3}, H^{4} \leq H^{3}, h^{4} \leq h^{3}$ such that if

$$
\begin{gathered}
p^{4}=z^{0} \frown\left\langle w, A^{4}, H^{4}, h^{4}\right\rangle, \\
z^{\prime} \frown X \frown\left\langle w, A^{\prime}, H^{\prime}, h^{\prime}\right\rangle \leq p^{4}
\end{gathered}
$$

is a condition deciding $\beta, \operatorname{lh}\left(u_{X}\right)>1$, and $\kappa_{X}>\kappa_{z^{0}}$ then for some $\beta$ and for $w(\beta)$-many $u$

$$
\exists B \quad \exists h z^{\prime} \frown\left\langle u, B, H^{4}\left\lceil\left[\kappa_{u}\right]^{2}, h\right\rangle \frown\left\langle w, A^{4}, H^{4}, H^{4}\left(\kappa_{u}\right)\right\rangle \| \beta .\right.
$$

Construction. For each lower part $x$ and $\beta<\operatorname{lh}(w)$ let

$$
\begin{aligned}
& X^{x, 1}=\left\{\langle\alpha\rangle \mid\langle\alpha\rangle \in A^{3}\right\}, \\
& X_{0}^{x, \beta}=\left\{v \mid \exists B \quad \exists I \exists i \quad x \frown\langle v, B, I, i\rangle \frown\left\langle w, A^{3}, H^{3}, H^{3}\left(\kappa_{v}\right)\right\rangle \| \beta\right\}, \\
& X_{1}^{x, \beta}=\left(X_{0}^{x, \beta}\right)^{c}, \\
& X^{x, \beta}=X_{i}^{x, \beta} \quad \text { for the unique } i \text { such that } X_{i}^{x, \beta} \in w(\beta), \quad \beta>1 .
\end{aligned}
$$

Now for each $x$ let

$$
X^{x}=\bigcup_{0<\beta<\operatorname{lh}(w)} X^{x, \beta}
$$

and define

$$
X=\left\{v \mid \forall x \kappa_{x}<\kappa_{v} \Rightarrow v \in X^{x}\right\} .
$$

The point of this definition is that $X \in \mathscr{F}_{w}$, and if $v \in X, \ln (v)>1$, and

$$
\exists B \quad \exists I \exists i x \frown\langle v, B, I, i\rangle \frown\left\langle w, A^{3}, H^{3}, H^{3}\left(\kappa_{v}\right)\right\rangle \| \beta
$$

then for some $\beta<\ln (w)$ the relation $(*)$ holds for $w(\beta)$-many $v$.

For each $x$, if there exists such a $v$, find one such that the value of $\beta$ associated with it is minimal. By the definition of $w(\beta)$ there are $B_{x}, I_{x}, i_{x}$ in $k(V)$ :

$$
x \frown\left\langle w \mid \beta, B_{x}, I_{x}, i_{x}\right\rangle \frown\left\langle k(w), k\left(A^{3}\right), k\left(H^{3}\right), k\left(H^{3}\right)\left(\kappa_{w}\right)\right\rangle \| k(b) .
$$

Let $I \in g^{* *}$ be such that

$$
\forall x \quad I \leq^{*} I_{x}
$$

and find $C \in \mu_{w}$ such that

$$
\forall(\alpha, \beta) \in[C]^{2} \forall x \quad \kappa_{x}<\alpha<\beta \Rightarrow I(\alpha, \beta) \leq I_{x}(\alpha, \beta) .
$$

Define $A^{4} \subset A^{3} \cap\left\{u \mid \kappa_{u} \in C\right\}, H^{4} \leq H^{3}, I$, and $h^{4} \leq h^{3}$ appropriately. By construction $p^{4}$ has the right property.

Step Five. We find $A^{5} \subseteq A^{4}, H^{5} \leq H^{4}, h^{5} \leq h^{4}$ such that if

$$
p^{5}={ }_{\text {def }} z^{0} \frown\left\langle w, A^{5}, H^{5}, h^{5}\right\rangle
$$

and

$$
z^{\prime} \frown\left\langle v, A, H^{5} \uparrow\left[\kappa_{v}\right]^{2}, H^{5}\left(\kappa_{z^{\prime}}\right) \mid \kappa_{v}\right\rangle \frown\left\langle w, A^{5}, H^{5}, H^{5}\left(\kappa_{v}\right)\right\rangle \leq p^{5},
$$

$\operatorname{lh}(v)>1, \kappa_{v}>\kappa_{z^{0}}$, is a condition which forces $b$ then for some $\beta$

$$
\exists A z^{\prime} \frown\left\langle v, A, H^{5}\left\lceil\left[\kappa_{v}\right]^{2}, H^{5}\left(\kappa_{z^{\prime}}\right)\left\lceil\kappa_{v}\right\rangle \frown\left\langle w, A^{5}, H^{5}, H^{5}\left(\kappa_{v}\right)\right\rangle \| b\right.\right.
$$

for $w(\beta)$-many $v$ (and similarly for $\neg b$ ). 
Construction. Define $C^{x, 1}=\left\{\langle\alpha\rangle \mid\langle\alpha\rangle \in A^{4}\right\}$. Then for $1<\beta<\ln (w)$ let

$$
\begin{aligned}
B_{0}^{x}=\{v \mid \exists A x \frown\langle v, A, & H^{4}\left\lceil\left[\kappa_{v}\right]^{2}, H^{4}\left(\kappa_{x}\right)\left\lceil\kappa_{v}\right\rangle\right. \\
& \left.\frown\left\langle w, A^{4}, H^{4}, H^{4}\left(\kappa_{v}\right)\right\rangle \Vdash b\right\}, \\
B_{1}^{x}=\{v \mid \exists A x \frown\langle v, A, & H^{4}\left\lceil\left[\kappa_{v}\right]^{2}, H^{4}\left(\kappa_{x}\right)\left\lceil\kappa_{v}\right\rangle\right. \\
& \left.\frown\left\langle w, A^{4}, H^{4}, H^{4}\left(\kappa_{v}\right)\right\rangle \Vdash \neg b\right\}, \\
B_{2}^{x}=\{v \mid \exists A x \frown\langle v, A, & H^{4}\left\lceil\left[\kappa_{v}\right]^{2}, H^{4}\left(\kappa_{x}\right)\left\lceil\kappa_{v}\right\rangle\right. \\
& \left.\frown\left\langle w, A^{4}, H^{4}, H^{4}\left(\kappa_{v}\right)\right\rangle \nmid b\right\},
\end{aligned}
$$$$
C^{x, \beta}=B_{i}^{x} \quad \text { for the unique } i \text { such that } B_{i}^{x} \in w(\beta) .
$$

Finally define

$$
C^{x}=\bigcup_{0<\beta<\operatorname{lh}(w)} C^{x, \beta}, \quad C=\left\{v \mid \forall x \kappa_{x}<\kappa_{v} \Rightarrow v \in C^{x}\right\} .
$$

Let $A^{5}=A^{4} \cap C$ and refine $H^{4}, h^{4}$ appropriately.

Step Six. We find $A^{6} \subseteq A^{5}, H^{6} \leq H^{5}, h^{6} \leq h^{5}$ such that if

$$
\begin{gathered}
p^{6}=z^{0} \frown\left\langle w, A^{6}, H^{6}, h^{6}\right\rangle, \\
z^{\prime} \frown\left\langle H^{6}\left(\kappa_{z^{\prime}}, \beta\right), \beta\right\rangle \frown\left\langle w, A^{6}, H^{6}, H^{6}(\beta)\right\rangle \leq p^{6}
\end{gathered}
$$

forces $b$, and $\beta>\kappa_{z^{0}}$, then

$$
z^{\prime} \frown\left\langle H^{6}\left(\kappa_{z^{\prime}}, \gamma\right), \gamma\right\rangle \frown\left\langle w, A^{6}, H^{6}, H^{6}(\gamma)\right\rangle \Vdash b
$$

for all $\gamma$ with $\langle\gamma\rangle \in A^{6}$. Notice that this implies that

$$
z^{\prime} \frown\left\langle w, A^{6}, H^{6}, H^{6}\left(\kappa_{z^{\prime}}\right)\right\rangle \Vdash b
$$

as any extension of this condition is comparable with a condition forcing $b$. (Of course the same claim is also true with $\neg b$ in the place of $b$.)

Construction.

$$
\begin{aligned}
B_{0}^{x} & =\left\{\beta \mid x \frown\left\langle H^{5}\left(\kappa_{x}, \beta\right), \beta\right\rangle \frown\left\langle w, A^{5}, H^{5}, H^{5}(\beta)\right\rangle \Vdash b\right\}, \\
B_{1}^{x} & =\left\{\beta \mid x \frown\left\langle H^{5}\left(\kappa_{x}, \beta\right), \beta\right\rangle \frown\left\langle w, A^{5}, H^{5}, H^{5}(\beta)\right\rangle \Vdash \neg b\right\}, \\
B_{2}^{x} & =\left\{\beta \mid x \frown\left\langle H^{5}\left(\kappa_{x}, \beta\right), \beta\right\rangle \frown\left\langle w, A^{5}, H^{5}, H^{5}(\beta)\right\rangle \nVdash b\right\}, \\
B^{x} & =B_{i}^{x} \quad \text { for the unique } i \text { with } B_{i}^{x} \in \mu_{w}, \\
B & =\left\{v \mid \forall x \kappa_{x}<\kappa_{v} \Rightarrow \kappa_{v} \in B^{x}\right\} .
\end{aligned}
$$

Let $A^{6}=A^{5} \cap B$ and define $H^{6}, h^{6}$ by an appropriate shrinking of the domains of $H^{5}, h^{5}$. This finishes the construction phase of the proof.

Step Seven. We prove that $p^{6}$ is an extension which decides $b$ "up to lower parts." That is, $b$ can be decided by an extension in which no object $X$ with $\kappa_{X}>\kappa_{z^{0}}$ is inserted.

Proof. Find an extension $r$ of $p^{6}$ deciding $b$ such that the lower part $x$ has a minimal number of entries $Y$ with $\kappa_{Y}>\kappa_{z^{0}}$, and such that among such conditions $\kappa_{x}$ has the minimal value. 
Claim 1. $\kappa_{x}=\kappa_{z^{0}}$ (i.e, the minimal number of entries $Y$ with $\kappa_{Y}>\kappa_{z^{0}}$ is in fact zero).

Proof of Claim 1. Suppose that $\kappa_{x}>\kappa_{z^{0}}$, and let $x=z^{\prime} \frown X$, where $X$ is a single entry. By the work done in Steps One and Two

$$
z^{\prime} \frown X \frown\left\langle w, A^{6}, H^{6}, H^{6}\left(\kappa_{X}\right)\right\rangle \| b .
$$

Suppose that in fact it forces $b$ (the argument for $\neg b$ will be identical).

Subclaim $1 . X$ is a pair, rather than a quadruple.

Proof of Subclaim 1. Let $X$ be a quadruple $\langle v, B, I, i\rangle$. The construction of Step Five guarantees

$$
\left\{v \mid \exists B \exists i z^{\prime} \frown\left\langle v, B, H^{6} \mid\left[\kappa_{v}\right]^{2}, i\right\rangle \frown\left\langle w, A^{6}, H^{6}, H^{6}\left(\kappa_{v}\right)\right\rangle \Vdash b\right\} \in w(\beta)
$$

for some $\beta>1$. For each such $v$ choose appropriate $B, i$, and let $\langle P, \alpha\rangle$ be some pair such that $\langle\alpha\rangle \in B$ and $P \leq i(\alpha)$. An easy normality argument ( $v \mapsto \alpha<\kappa_{v}$, so fix $\alpha$ on a large set; now use completeness to fix $P$ ) gives that the choice of $\langle P, \alpha\rangle$ can be stabilised on a set in $w(\beta)$. Fix some $\langle P, \alpha\rangle$ such that for $w(\beta)$-many $v$ there exists $B$ :

$$
z^{\prime} \frown\langle P, \alpha\rangle \frown\left\langle v, B, H^{6}\left\lceil\left[\kappa_{v}\right]^{2}, H^{6}(\alpha)\left|\kappa_{v}\right\rangle \frown\left\langle w, A^{6}, H^{6}, H^{6}\left(\kappa_{v}\right)\right\rangle \Vdash b .\right.\right.
$$

Fix $f$ a choice function giving for each such $v$ an appropriate $B$. The situation is now essentially the same as that in the last stage of the proof that ordinary Radin forcing has the Prikry property (see $[R]$ ). Define sets

$$
\begin{aligned}
& A_{1}^{0}=\{v \mid\{t \in \operatorname{dom}(f) \mid v \in f(t)\} \in w(\beta)\}, \\
& A_{1}^{1}=\left\{v \mid\left\{t \in \operatorname{dom}(f) \mid f(t) \cap V_{\kappa_{v}} \in \mathscr{F}_{v}\right\} \in w(\beta)\right\}, \\
& A^{1}=A_{1}^{0} \cap A_{1}^{1}, \\
& A_{2}=\left\{t \in \operatorname{dom}(f) \mid \forall v \in A_{1} \quad \kappa_{v}<\kappa_{t} \Rightarrow v \in f(t)\right\}, \\
& A_{3}=\left\{v \mid \forall C \in \mathscr{F}_{v} \quad \exists \mu<\operatorname{lh}(v)\left\{t \in A^{2} \cap C \mid f(t) \cap C \in \mathscr{F}_{v}\right\} \in v(\mu)\right\} .
\end{aligned}
$$

Using the constructing pair argument it is routine to check that

(1) $A_{1} \in \mathscr{F}_{w\lceil\beta}$.

(2) $A_{2} \in w(\beta)$.

(3) $A_{3} \in \bigcap\{w(\alpha) \mid \beta<\alpha<\ln (w)\}$.

So $A_{1} \cup A_{2} \cup A_{3} \in \mathscr{F}_{w}$. Define $A^{\prime}=A^{6} \cap\left(A_{1} \cup A_{2} \cup A_{3}\right)$. The following subclaim gives us what we need.

Subclaim 2. Any extension of

$$
z^{\prime} \frown\langle P, \alpha\rangle \frown\left\langle w, A^{\prime}, H^{6}, H^{6}(\alpha)\right\rangle
$$

is comparable with

$$
z^{\prime} \frown\langle P, \alpha\rangle \frown\left\langle v, f(v), H^{6}\right|\left[\kappa_{v}\right]^{2}, H^{6}(\alpha)\left\lceil\kappa_{v}\right\rangle \frown\left\langle w, A^{6}, H^{6}, H^{6}\left(\kappa_{v}\right)\right\rangle
$$

for some $v \in \operatorname{dom}(f)$.

Proof of Subclaim 2. Let the extension be

$$
p=\bar{z} \frown X_{1} \frown X_{2} \frown \cdots \frown X_{n} \frown\left\langle w, A^{*}, H^{*}, h^{*}\right\rangle,
$$


where $\kappa_{\bar{z}}=\alpha . \forall i \quad \exists j \quad u_{X_{i}} \in A_{j}$. Let $v$ be the first sequence with $v \notin A_{1}$. Then $v \in A_{2}, v \in A_{3}$, or $v=w$.

Case (1). $v \in A_{2}$. Say $v=u_{X_{j}}$. By construction

$$
v \in \operatorname{dom}(f), \quad \forall i<j \quad f(v) \cap V_{\kappa_{X_{i}}} \in \mathscr{F}_{u_{X_{i}}} .
$$

So defining (for $i \leq j$ )

$$
X_{i}^{\prime}= \begin{cases}\langle t, A \cap f(v), H, h\rangle & \text { if } X_{i}=\langle t, A, H, h\rangle, \\ \langle Q, \delta\rangle & \text { if } X_{i}=\langle Q, \delta\rangle,\end{cases}
$$

the condition

$$
\bar{z} \frown X_{1}^{\prime} \frown \cdots \frown X_{j}^{\prime} \frown X_{j+1} \frown \cdots \frown X_{n} \frown\left\langle w, A^{*}, H^{*}, h^{*}\right\rangle
$$

is a common extension of $p$ and

$$
z^{\prime} \frown\langle P, \alpha\rangle \frown\left\langle v, f(v), H^{6}\left\lceil\left[\kappa_{v}\right]^{2}, H^{6}(\alpha)\left\lceil\kappa_{v}\right\rangle \frown\left\langle w, A^{6}, H^{6}, H^{6}\left(\kappa_{u}\right)\right\rangle .\right.\right.
$$

Case (2). $v=u_{X_{j}} \in A_{3}$. Say $X_{j}=\langle v, A, H, h\rangle$. By construction find $v^{\prime}$ with $\kappa_{v^{\prime}}>\kappa_{X_{j-1}}, v^{\prime} \in A \cap A_{2}$, and $f\left(v^{\prime}\right) \cap A \in \mathscr{F}_{v^{\prime}}$. As before $\forall i<j f\left(v^{\prime}\right) \cap V_{\kappa_{X_{i}}}$ $\in \mathscr{F}_{u_{X_{i}}}$, so defining $X_{i}^{\prime}$ as before for $i<j$

$$
\begin{gathered}
\bar{z} \frown X_{1}^{\prime} \frown \cdots \frown X_{j-1}^{\prime} \frown\left\langle v^{\prime}, f\left(v^{\prime}\right), H^{6} \uparrow\left[\kappa_{v^{\prime}}\right]^{2}, H^{6}\left(\kappa_{X_{j-1}}\right) \uparrow \kappa_{v^{\prime}}\right\rangle \\
\frown X_{j} \frown \cdots \frown X_{n} \frown\left\langle w, A^{*}, H^{*}, h^{*}\right\rangle
\end{gathered}
$$

is the sought-after common extension.

Case (3). Like Case (2). Exploit the fact that

$$
\left\{v \in A^{2} \mid f(v) \cap B^{*} \in \mathscr{F}_{w}\right\} \in w(\beta)
$$

to produce an appropriate $v^{\prime}$ with $\kappa_{v^{\prime}}>\kappa_{X_{n}}$. The subclaim is proved.

So the condition

$$
r^{\prime}=z^{\prime} \frown\langle P, \alpha\rangle \frown\left\langle w, A^{\prime}, H^{6}, H^{6}(\alpha)\right\rangle
$$

has the property that any extension of it is compatible with some condition which forces $b$. Thus by elementary facts about forcing the condition itself forces $b$. However this contradicts the assumption that $r$ was chosen to minimise the last ordinal mentioned in the lower part, amongst conditions extending $p^{5}$, deciding $b$, and having the same number of entries $Y$ with $\kappa_{Y}>\kappa_{z^{0}}$ as does $x$. The subclaim is therefore proved.

If $\kappa_{x}>\kappa_{z^{0}}$ then by the subclaim $x=z^{\prime} \frown\langle P, \beta\rangle$, where $\kappa_{z^{\prime}}=\kappa_{z^{0}}$. For if not then

$$
z^{\prime} \frown\left\langle H\left(\kappa_{z^{\prime}}, \beta\right), \beta\right\rangle \frown\left\langle w, A^{6}, H^{6}, H^{6}(\beta)\right\rangle \Vdash b
$$

by the work done in Step Three. Then

$$
z^{\prime} \frown\left\langle w, A^{6}, H^{6}, H^{6}\left(\kappa_{z^{\prime}}\right)\right\rangle \Vdash b
$$

by Step Six. This contradicts the choice of $x$ to contain as few entries past $\kappa_{z^{0}}$ as possible.

Now define

$$
C=\left\{\beta \mid \exists z_{\beta} \exists P \leq h^{6}(\beta) z_{\beta} \frown\langle P, \beta\rangle \frown\left\langle w, A^{6}, H^{6}, H^{6}(\beta)\right\rangle \| b\right\} .
$$


Subclaim 3. $C \in \mu_{w}$.

Proof. Suppose not, and let $A^{*}=A^{6} \backslash\left\{w \mid \kappa_{w} \in C\right\}$, define $H^{*}, h^{*}$ by restriction of $H^{6}, h^{6}$, and argue as above that extension of the condition $z^{0} \frown\left\langle w, A^{*}, H^{*}, h^{*}\right\rangle$ in the same "minimal" way to decide $b$ demands adding at worst one pair $Y$ with $\kappa_{Y}>\kappa_{z^{0}}$; this gives an immediate contradiction.

Pick for each $\beta \in C$ an appropriate $z_{\beta}$. Find $C^{\prime} \in \mu_{w}$ on which $z_{\beta}$ is constant, with value $z^{*}$ say, and such that every element chosen decides $b$ the same way. Shrinking $A^{6}$ to

defining $h^{\prime}$ so that for each $\beta$ in $C$

$$
A^{\prime}=A^{6} \cap\left\{w \mid \kappa_{w} \in C^{\prime}\right\},
$$

$$
z^{*} \frown\left\langle h^{\prime}(\beta), \beta\right\rangle \frown\left\langle w, A^{6}, H^{6}, H^{6}(\beta)\right\rangle \| b,
$$

and shrinking $H^{6}$ to $H^{\prime}$ appropriately the proof is done. The condition $z^{*} \frown\left\langle w, A^{6}, H^{6}, h^{\prime}\right\rangle$ decides $b$.

Step Eight. We conclude the proof by showing that $b$ can be decided by a direct extension of $p^{0}$.

Proof. We just use induction on the length of conditions and the following obvious fact about the forcing $Q_{w}$.

Fact 1. If $p \in Q_{w}$ is a condition of the form

$$
x \frown\langle v, B, I, i\rangle \frown\left\langle P_{0}, \delta_{0}\right\rangle \cdots\left\langle P_{n}, \delta_{n}\right\rangle \frown\langle w, A, H, h\rangle
$$

then $Q_{w} \uparrow p$ factors as $P_{1} \times P_{2} \times P_{3}$, where

(1) $P_{1}=Q_{v}\lceil x \frown\langle v, B, I, i\rangle$.

(2) $P_{2}=P\left(\kappa_{v_{0}}, \delta_{0}\right)\left\lceil P_{0} \times \cdots \times P\left(\delta_{n-1}, \delta_{n}\right) \uparrow P_{n}\right.$.

(3) $P_{3}=Q_{w} \mid\left\langle\delta_{n}\right\rangle \frown\langle w, A, H, h\rangle$.

The theorem is proved.

Using this theorem we can go some way towards showing that the cardinals of the extension by $Q_{w}$ are exactly those which we have not taken steps to collapse.

Theorem 2. Let $\vec{v}, \vec{F}$ be $Q_{w}$-generic and as usual define $\kappa_{\alpha}=\kappa_{v(\alpha)}$. Then for every $\alpha$ the $V$-cardinals $\kappa_{\alpha}, \kappa_{\alpha}^{++}, \kappa_{\alpha}^{+3}, \kappa_{\alpha}^{+4}$, are cardinals in the generic extension.

Proof. We divide the proof into two cases.

Case (1) $\alpha$ is a limit. One application of the theorem shows that if $X$ is a bounded subset of $\kappa_{\alpha}$ in the extension then $X$ is generic for forcing of cardinality less than $\kappa_{\alpha}$; hence $\kappa_{\alpha}$ survives as a cardinal. Similarly if $X$ is a bounded subset of $\kappa_{\alpha}^{+4}$ then $X$ is in the extension by $Q_{v_{\alpha}}$, which is $\kappa_{\alpha}^{++}$-c.c. and therefore preserves $\kappa_{\alpha}^{+2}, \kappa_{\alpha}^{+3}, \kappa_{\alpha}^{+4}$.

Case (2) $\alpha$ is a successor. Say $\alpha=\lambda+n$ for $\lambda$ limit. As before it is easy to argue that $\kappa_{\alpha}$ is preserved. Now we have that bounded subsets of $\kappa_{\alpha}^{+4}$ are in the extension by

$$
Q_{v(\lambda)} \times P\left(\kappa_{\lambda}, \kappa_{\lambda+1}\right) \times \cdots \times P\left(\kappa_{\lambda+n-1}, \kappa_{\alpha}\right),
$$

which is $\kappa_{\alpha}^{++}$-c.c. forcing. The theorem is proved. 
Of course we have explicitly collapsed $\kappa_{\alpha}^{+}$for $\alpha$ successor, so the question left open here is the fate of $\kappa_{\alpha}^{+}$when $\alpha$ is limit. The answer to this must await the fine analysis of $Q_{w}$-genericity which we undertake in the next subsection.

We also make the remark that the same kind of analysis will show that we have not added too many subsets to any cardinal of the extension; in fact, once we have shown that $\kappa_{\alpha}^{+}$is preserved for $\alpha$ limit it will be easy to show that in the extension GCH holds at all successors and fails at all limits.

3.5. The geometric condition. We prove a simple criterion for $Q_{w}$ genericity, analogous to Mathias' criterion for Prikry genericity [M]. Recall that if $(\vec{u}, \vec{F})$ is generic with $\ln (\vec{u})=\ln (\vec{F})=\lambda$ then

(a) If $\mu<\lambda$ is such that $\ln \left(u_{\mu}\right)>1$ then the pair $(\vec{u}\lceil\mu, \vec{F}\lceil\mu)$ is generic for the forcing $Q_{u_{\mu}}$.

(b) $\forall A \in V_{\kappa_{w}+1}\left(A \in \mathscr{F}_{w} \Leftrightarrow \exists \beta \quad \forall \alpha>\beta \quad u_{\alpha} \in A\right)$.

(c) $\forall H \in w(1) \exists \beta \quad \forall \alpha>\beta H\left(\kappa_{\alpha}, \kappa_{\alpha+1}\right) \in F_{\alpha}$.

Theorem 1. The pair $\left(\left\langle u_{\alpha}: \alpha<\lambda\right\rangle,\left\langle F_{\alpha}: \alpha<\lambda\right\rangle\right)$ is generic if and only if it satisfies conditions (a), (b), (c).

Proof.

Lemma 1. Let $D$ be an open set, and let $A, H, \beta$ be such that $D$ is dense below $\langle\beta\rangle \frown\langle w, A, H, H(\beta)\rangle$. By the methods of the Prikry property proof, Steps One and Two, it may be assumed that if

$$
x \frown\left\langle w, A^{\prime}, H^{\prime}, h^{\prime}\right\rangle \leq\langle\beta\rangle \frown\langle w, A, H, H(\beta)\rangle
$$

is a member of $D$ and $\kappa_{x}>\beta$ then

$$
x \frown\left\langle w, A, H, H\left(\kappa_{x}\right)\right\rangle \in D .
$$

Then there exists $j<\omega$ such that (introducing a quantifier $\forall_{\mu}^{*}$, where $\forall_{\mu}^{*} x \phi(x)$ is to be interpreted as " $\phi(x)$ holds on a set in the ultrafilter $\mu$ ")

$$
\begin{aligned}
& \exists n_{0} \forall_{U^{n_{0}}}^{*}\left(\delta_{1}^{0}, \ldots, \delta_{n_{0}}^{0}\right) \exists\left(P_{1}^{0}, \ldots, P_{n_{0}}^{0}\right) \exists \beta_{0}>1 \forall_{w\left(\beta_{0}\right)}^{*} u_{0} \exists A_{0} \exists H_{0} \\
& \ldots \\
& \exists n_{j} \forall_{U^{n_{j}}}^{*}\left(\delta_{1}^{j}, \ldots, \delta_{n_{j}}^{j}\right) \exists\left(P_{1}^{j}, \ldots, P_{n_{j}}^{j}\right) \exists \beta_{j}>1 \forall_{w\left(\beta_{j}\right)}^{*} u_{j} \exists A_{j} \exists H_{j} \\
& \langle\beta\rangle \frown\left\langle P_{1}^{0}, \delta_{1}^{0}\right\rangle \frown \cdots \frown\left\langle P_{n_{0}}^{0}, \delta_{n_{0}}^{0}\right\rangle \frown\left\langle u_{0}, A_{0}, H_{0}, H_{0}\left(\delta_{n_{0}}^{0}\right)\right\rangle \\
& \quad \frown \cdots \frown\left\langle P_{1}^{j}, \delta_{1}^{j}\right\rangle \frown \cdots \frown\left\langle P_{n_{j}}^{j}, \delta_{n_{j}}^{j}\right\rangle \frown\left\langle u_{j}, A_{j}, H_{j}, H_{j}\left(\delta_{n_{j}}^{j}\right)\right\rangle \\
& \quad \frown\left\langle w, A, H, H\left(\kappa_{u_{j}}\right)\right\rangle
\end{aligned}
$$

extends $\langle\beta\rangle \frown\langle w, A, H, H(\beta)\rangle$ and is in $D$.

Proof of Lemma 1. Suppose not. For the sake of brevity abbreviate the formula

$$
\begin{aligned}
“\langle\beta\rangle \frown\left\langle P_{1}^{0}, \delta_{1}^{0}\right\rangle \frown \cdots \frown\left\langle P_{n_{0}}^{0}, \delta_{n_{0}}^{0}\right\rangle \frown\left\langle u_{0}, A_{0}, H_{0}, H_{0}\left(\delta_{n_{0}}^{0}\right)\right\rangle \\
\frown \cdots \frown\left\langle P_{1}^{j}, \delta_{1}^{j}\right\rangle \frown \cdots \frown\left\langle P_{n_{j}}^{j}, \delta_{n_{j}}^{j}\right\rangle \frown\left\langle u_{j}, A_{j}, H_{j}, H_{j}\left(\delta_{n_{j}}^{j}\right)\right\rangle \\
\frown\left\langle w, A, H, H\left(\kappa_{u_{j}}\right)\right\rangle
\end{aligned}
$$


extends $\langle\beta\rangle \frown\langle w, A, H, H(\beta)\rangle$ and is in $D$ " to $\phi_{j}(\vec{\delta}, \vec{P}, \vec{w}, \vec{A}, \vec{H})$, and the quantifier sequence

$$
\forall n_{i} \forall_{U^{n_{i}}}^{*}\left(\delta_{1}^{i}, \ldots, \delta_{n_{i}}^{i}\right) \forall\left(P_{1}^{i}, \ldots, P_{n_{i}}^{i}\right) \forall \beta_{i}>1 \forall_{w\left(\beta_{i}\right)}^{*} u_{i} \forall A_{i} \forall H_{i}
$$

to $Q_{i}$. So for all $j$

$$
Q_{0} \quad Q_{1} \cdots Q_{j} \neg \phi_{j}(\vec{\delta}, \vec{P}, \vec{w}, \vec{A}, \vec{H}) .
$$

Fix $j$ and $i \leq j$ for the moment, and define

$$
\begin{aligned}
& Y_{i, j}^{s}=\left\{u_{i} \in A^{\prime} \mid \operatorname{lh}\left(u_{i}\right)=1 \vee \forall A_{i} \forall H_{i} \quad Q_{i+1} \cdots Q_{j} \neg \phi_{j}(\vec{\delta}, \vec{P}, \vec{w}, \vec{A}, \vec{H})\right\}, \\
& Y_{i, j}=\left\{u \mid \forall s \delta_{n_{i}}^{i}<\kappa_{u} \Rightarrow u \in Y_{i, j}^{s}\right\}
\end{aligned}
$$

for each

$$
s=\left\langle\delta_{1}^{0}, \ldots, \delta_{n_{0}}^{0}, P_{1}^{0}, \ldots, P_{n_{0}}^{0}, u_{0}, A_{0}, h_{0}, \ldots, P_{1}^{i}, \ldots, P_{n_{i}}^{i}\right\rangle
$$

such that

$$
\forall \beta_{i}>1 \quad \forall_{w\left(\beta_{i}\right)}^{*} u_{i} \forall A_{i} \quad \forall H_{i} \quad Q_{i+1} \cdots Q_{j} \neg \phi_{j}(\vec{\delta}, \vec{P}, \vec{w}, \vec{A}, \vec{H}) .
$$

Now let $i, j$ vary and define

$$
Y=\operatorname{def} \bigcap_{j<\omega} \bigcap_{i<\omega} Y_{i, j} .
$$

For each $t=\left\langle\delta_{1}^{0}, \ldots, A_{i}, H_{i}\right\rangle$ such that

$$
\forall n_{i+1} \quad \forall_{U^{n_{i+1}}}\left\langle\delta_{1}^{i+1}, \ldots, \delta_{n_{i+1}}^{i+1}\right\rangle \cdots \forall H_{i+1} \quad Q_{i+2} \cdots Q_{j} \neg \phi(\vec{\delta}, \vec{P}, \vec{w}, \vec{A}, \vec{H})
$$

define $Z^{t, n_{i+1}}$ for each $n_{i+1}$ to be

$$
\left\{\vec{\delta}^{i+1} \in[\kappa]^{n_{i+1}} \mid \forall \vec{P}^{i+1} \quad \forall H_{i+1} \quad Q_{i+2} \cdots Q_{j} \neg \phi(\vec{\delta}, \vec{P}, \vec{w}, \vec{A}, \vec{H})\right\}
$$

and find $X^{t} \in U$ such that $\forall n_{i+1}\left[X^{t}\right]^{n_{i+1}} \subset Z^{t, n_{i+1}}$. Find $Y^{\prime} \subset Y, Y^{\prime} \in \mathscr{F}_{w}$ such that $\langle\alpha\rangle \in Y^{\prime} \wedge \operatorname{rank}(t)<\alpha \Rightarrow \alpha \in X^{t}$ and $\langle\beta\rangle \frown\langle w, Y, H, H(\beta)\rangle$ has some extension in $D$, say

$$
\begin{gathered}
y_{0} \frown\left\langle u_{0}, A_{0}, H_{0}, h_{0}\right\rangle \frown \cdots \frown y_{n} \frown\left\langle u_{n}, A_{n}, H_{n}, h_{n}\right\rangle \\
\frown y_{n+1} \frown\left\langle w, Y, H, H\left(\kappa_{y_{n+1}}\right)\right\rangle \in D .
\end{gathered}
$$

As $D$ is open it is possible to extend this condition to

$$
\begin{aligned}
y_{0} \frown\left\langle u_{0}, A_{0}, H_{0}, h_{0}\right\rangle \frown \cdots \frown y_{n} \frown\left\langle u_{n}, A_{n}, H_{n}, h_{n}\right\rangle \\
\quad \frown y_{n+1} \frown\left\langle u_{n+1}, A_{n+1}, H_{n+1}, h_{n+1}\right\rangle \frown\left\langle w, Y, H, H\left(\kappa_{u_{n+1}}\right)\right\rangle \in D,
\end{aligned}
$$

contradicting the definition of $Y^{\prime}$.

Fix $j$ with the property guaranteed by the lemma above. Using the same idea as in Step Three of the proof of the Prikry property refine $A, H$ to $A^{*}$, 
$H^{*}$ such that

$$
\begin{aligned}
& \exists n_{0} \forall_{U^{n_{0}}}^{*}\left(\delta_{1}^{0}, \ldots, \delta_{n_{0}}^{0}\right) \exists \beta_{0}>1 \forall_{w\left(\beta_{0}\right)}^{*} u_{0} \exists A_{0} \in \mathscr{F}_{u_{0}} \exists H_{0} \in h_{u_{0}} \\
& \ldots \\
& \exists n_{j} \forall_{U^{n_{j}}}^{*}\left(\delta_{1}^{j}, \ldots, \delta_{n_{j}}^{j}\right) \exists \beta_{j}>1 \forall_{w\left(\beta_{j}\right)}^{*} u_{j} \exists A_{j} \in \mathscr{F}_{u_{j}} \exists H_{j} \in h_{u_{j}} \\
& \langle\beta\rangle \frown\left\langle H^{*}\left(\beta, \delta_{1}^{0}\right), \delta_{1}^{0}\right\rangle \frown \cdots \frown\left\langle H^{*}\left(\delta_{n_{0}-1}^{0}, \delta_{n_{0}}^{0}\right), \delta_{n_{0}}^{0}\right\rangle \\
& \quad \frown\left\langle u_{0}, A_{0}, H_{0}, H_{0}\left(\delta_{n_{0}}^{0}\right)\right\rangle \frown \cdots \frown\left\langle H^{*}\left(\kappa_{u_{j-1}}, \delta_{1}^{j}\right), \delta_{1}^{j}\right\rangle \\
& \quad \frown \cdots \frown\left\langle H^{*}\left(\delta_{n_{j}-1}^{j}, \delta_{n_{j}}^{j}\right), \delta_{n_{j}}^{j}\right\rangle \\
& \quad \frown\left\langle u_{j}, A_{j}, H_{j}, H_{j}\left(\delta_{n_{j}}^{j}\right)\right\rangle \frown\left\langle w, A^{*}, H^{*}, H^{*}\left(\kappa_{u_{j}}\right)\right\rangle \\
& \text { extends }\langle\beta\rangle \frown\left\langle w, A^{*}, H^{*}, H^{*}(\beta)\right\rangle \text { and is in } D .
\end{aligned}
$$

Abbreviate this formula as

$$
R_{0} R_{1} \cdots R_{j} \psi(\vec{\delta}, \vec{w}, \vec{A}, \vec{H})
$$

At the same time arrange that the $\delta_{b}^{a}$ drawn from $A^{*}$ all come from appropriate measure one sets, as in the proof of the previous lemma.

Lemma 2. With the same hypotheses and notation as above there exist $A^{* *}, H^{* *}$ refining $A^{*}, H^{*}$, such that if $\left(\left\langle v_{\gamma}: \gamma<\mu\right\rangle,\left\langle G_{\gamma}: \gamma<\mu\right\rangle\right)$ satisfies conditions (a), (b), (c), $v_{0}=\langle\beta\rangle$, and in addition

$$
\forall \gamma\left\{\begin{array}{l}
v_{\gamma} \in A^{* *}, \\
\left(\kappa_{v_{\gamma}}, \kappa_{v_{\gamma+1}}\right) \in \operatorname{dom}\left(H^{* *}\right), \\
H^{* *}\left(\kappa_{v_{\gamma}}, \kappa_{v_{\gamma+1}}\right) \in G_{\gamma},
\end{array}\right.
$$

then $D$ meets the filter on $Q_{w}$ generated by $\left(\left\langle v_{\gamma}: \gamma<\mu\right\rangle,\left\langle G_{\gamma}: \gamma<\mu\right\rangle\right)$.

Proof. For each

$$
t=\left\langle\delta_{1}^{0}, \ldots, \delta_{n_{0}}^{0}, u_{0}, A_{0}, H_{0}, \ldots, \delta_{1}^{i}, \ldots, \delta_{n_{i}}^{i}\right\rangle
$$

such that

$$
\exists \beta_{i}>1 \forall_{w\left(\beta_{i}\right)}^{*} u_{i} \exists A_{i} \in \mathscr{F}_{u_{i}} \exists H_{i} \in h_{w_{i}} R_{i+1} \cdots R_{j} \psi(\vec{\delta}, \vec{w}, \vec{A}, \vec{H})
$$

fix an appropriate $\beta^{t}$ and functions $f^{t}, g^{t}$ defined on a set $X^{t} \in w\left(\beta^{t}\right)$ such that

$$
\forall u_{i} \in X^{t} A_{i}=f^{t}\left(u_{i}\right) \wedge H_{i}=g^{t}\left(u_{i}\right) \Rightarrow R_{i+1} \cdots R_{j} \psi(\vec{\delta}, \vec{w}, \vec{A}, \vec{H})
$$

Now perform the following construction (which is reminiscent of the proof of a subclaim in the proof of the Prikry property, and indeed would prove a slightly stronger result in which " $H$-parts" are dependent on the sequence chosen in a 
more arbitrary way):

$$
\begin{aligned}
{ }^{t} A_{1}^{0}= & \left\{v \mid\{u \mid v \in f(u)\} \in w\left(\beta^{t}\right)\right\}=j(f)\left(w\left\lceil\beta^{t}\right),\right. \\
{ }^{t} A_{2}^{0}= & \left\{v \mid\left\{u \mid f(u) \cap V_{\kappa_{v}} \in \mathscr{F} v\right\} \in w\left(\beta^{t}\right)\right\} \\
= & \left\{v \mid A_{1}^{0} \cap V_{\kappa_{v}} \in \mathscr{F}_{v}\right\}, \\
{ }^{t} A_{3}^{0}= & \left\{v \mid\left\{u \mid g(u)\left\lceil\left[\kappa_{v}\right]^{2} \in h_{v}\right\} \in w\left(\beta^{t}\right)\right\}\right. \\
= & \left\{v \mid j(g)\left(w\left\lceil\beta^{t}\right)\left\lceil\left[\kappa_{v}\right]^{2} \in h_{v}\right\},\right.\right. \\
H^{t}= & j\left(g^{t}\right)\left(w\left\lceil\beta^{t}\right),\right. \\
{ }^{t} A^{0}= & \bigcup{ }^{t} A_{i}^{0}, \\
& 0<i<3 \quad \Rightarrow\left\{u \mid v \in f(u) \wedge f(u) \cap V_{\kappa_{v}}\right. \\
{ }^{t} A^{1}= & \left\{u \mid \forall v \in{ }^{t} A^{0} \kappa_{v}<\kappa_{u}\right. \\
& \left.\left.\quad \in \mathscr{F}_{v} \wedge g(u) \uparrow\left[\kappa_{v}\right]^{2} \in h_{v}\right\} \in w\left(\beta^{t}\right)\right\}, \\
{ }^{t} A^{2}= & \left\{x \mid \forall C \in \mathscr{F}_{x} \exists \mu<\operatorname{lh}(x) C \cap{ }^{t} A^{1} \in x(\mu)\right\} .
\end{aligned}
$$

As in the Prikry property proof it is easy to show

$$
\begin{aligned}
& { }^{t} A^{0} \in \mu_{w} \cap \bigcap_{1<\gamma<\beta^{t}} w(\gamma), \quad{ }^{t} A^{1} \in w\left(\beta^{t}\right), \\
& { }^{t} A^{3} \in \bigcap_{\beta^{t}<\gamma<\operatorname{lh}(w)} w(\gamma), \quad{ }^{t} A={ }_{\operatorname{def}} \bigcup_{i<2}{ }^{t} A^{i} \in \mathscr{F}_{w} .
\end{aligned}
$$

Define $A^{* *}, H^{* *}$ by taking the diagonal intersection of ${ }^{t} A, H^{t}$ over suitable $t$. To show that this construction works, select an $n_{0}$ such that

$$
\forall \vec{\delta}^{n_{0}} \in\left[\left\{\alpha \mid\langle\alpha\rangle \in A^{* *}\right\}\right]^{n_{0}} \quad \exists \beta_{0} \forall_{w\left(\beta_{0}\right)}^{*} u_{0} \quad \exists A_{0} H_{0} \quad R_{1} \cdots R_{j} \psi(\vec{\delta}, \vec{w}, \vec{A}, \vec{H}) .
$$

Define $\delta_{i}^{0}$ by $v_{i}=\left\langle\delta_{i}^{0}\right\rangle$ for $1 \leq i \leq n_{0}$. Set $t_{0}=\left\langle\delta_{1}^{0}, \ldots, \delta_{i}^{0}\right\rangle$. Find $\mu_{0}$ the least $\mu$ with $v_{\mu} \in{ }^{t_{0}} A^{1}$. The existence of such a $\mu$ is guaranteed by conditions (a), (b), (c), for if $\vec{v}$ avoids ${ }^{t_{0}} A$ on a final segment then by (b) $\left({ }^{t_{0}} A\right)^{c} \in \mathscr{F}_{w}$ which is absurd. Set $u_{0}=v_{\mu}$.

Claim 1. The condition

$$
\begin{aligned}
\langle\beta\rangle \frown & \left\langle H^{* *}\left(\beta, \delta_{1}^{0}\right), \delta_{1}^{0}\right\rangle \frown \cdots \frown\left\langle H^{* *}\left(\delta_{n_{0}-1}^{0}, \delta_{n_{0}}^{0}\right), \delta_{n_{0}}^{0}\right\rangle \\
& \frown\left\langle u_{0}, f^{t_{0}}\left(u_{0}\right), g^{t_{0}}\left(u_{0}\right), g^{t_{0}}\left(u_{0}\right)\left(\delta_{n_{0}}^{0}\right)\right\rangle \frown\left\langle w, A^{* *}, H^{* *}, H^{* *}\left(\kappa_{u_{0}}\right)\right\rangle
\end{aligned}
$$

is in the filter generated by $(\vec{v}, \vec{G})$.

Proof of Claim. It will suffice to show that for $\nu<\mu$

$$
v_{\nu} \in f^{t_{0}}\left(u_{0}\right), \quad g^{t_{0}}\left(u_{0}\right)\left(\kappa_{v_{\nu}}, \kappa_{v_{\nu+1}}\right) \in G_{\nu} \text {. }
$$

By construction $v_{\nu} \notin{ }^{t_{0}} A^{1}$. If $v_{\nu} \in{ }^{t_{0}} A^{0}$ then $v_{\nu} \in f^{t_{0}}\left(u_{0}\right)$ by the definition of ${ }^{t_{0}} A^{1}$. Suppose that $v_{\nu} \in{ }^{t_{0}} A^{2}$. Then for some $\sigma$

$$
f^{t_{0}}\left(v_{\nu}\right) \cap{ }^{t_{0}} A^{2} \in v_{\nu}(\sigma)
$$

but the sequence $\left\langle v_{\delta}: \delta\langle\nu\rangle\right.$ avoids this set, contradicting the local genericity condition (a). 
So $v_{\nu} \in f^{t_{0}}\left(u_{0}\right)$. To get the second clause of the claim notice that by construction

$$
H^{* *}\left(\kappa_{v_{\nu}}, \kappa_{v_{\nu+1}}\right) \leq g^{t_{0}}\left(u_{0}\right)\left(\kappa_{v_{\nu}}, \kappa_{v_{\nu+1}}\right)
$$

but $H^{* *}\left(\kappa_{v_{\nu}}, \kappa_{v_{\nu+1}}\right) \in G_{\nu}$ so $g^{t_{0}}\left(u_{0}\right)\left(\kappa_{v_{\nu}}, \kappa_{v_{\nu+1}}\right) \in G_{\nu}$. The claim follows.

Now select $n_{1}$ and so on; after $j$ stages this process will produce a condition in the filter which also lies in $D$.

Now fix $E$ a dense subset of $Q_{w}$. Let $x$ be a lower part of the form $z \frown\langle v, B, I, i\rangle$, i.e., a lower part which is a member of some $Q_{v}$.

If $q=\left\langle\kappa_{v}\right\rangle \frown y \frown\langle w, C, J, j\rangle$ then define

$$
x \oplus q=_{\operatorname{def}} x \frown y \frown\langle w, C, J, j\rangle .
$$

Let

$$
E_{x}=_{\text {def }}\left\{q \in Q_{w} \mid x \oplus q \in E\right\} .
$$

Fix $\nu$, and use the Prikry property to find $A_{\nu}, H_{\nu}$ such that

$$
\langle\nu\rangle \frown\left\langle w, A_{\nu}, H_{\nu}, H_{\nu}(\nu)\right\rangle \| \exists q x \oplus q \in E
$$

for each $x$ with $\kappa_{x}=\nu$. Say that $x$ is good or bad depending on whether $q$ is forced to exist or not. If $x$ is good it is easy to see that $E_{x}$ is dense below $\left\langle\kappa_{x}\right\rangle \frown\left\langle w, A_{\kappa_{x}}, H_{\kappa_{x}}, H_{\kappa_{x}}\left(\kappa_{x}\right)\right\rangle$. Apply Lemma 6.3 to produce appropriate $A_{x}^{* *}, H_{x}^{* *}$ for each good $x$, and define $A^{\dagger}, H^{\dagger}$ by taking the diagonal intersection over all good $x$. Because of (a), (b), (c) there is some $\beta<\lambda$ such that $\operatorname{lh}\left(u_{\beta}\right)>1$ and for all $\alpha>\beta$

$$
u_{\alpha} \in A^{\dagger}, \quad\left(\kappa_{u_{\alpha}}, \kappa_{u_{\alpha+1}}\right) \in \operatorname{dom}\left(H^{\dagger}\right), \quad H^{\dagger}\left(\kappa_{u_{\alpha}}, \kappa_{u_{\alpha+1}}\right) \in F_{\alpha} .
$$

Lemma 3. Good $x$ are dense in $Q_{u_{\beta}}$.

Proof. Fix $x \in Q_{u_{\beta}}$, and extend

$$
x \frown\left\langle w, A_{\kappa_{u_{\beta}}}, H_{\kappa_{u_{\beta}}}, H_{\kappa_{u_{\beta}}}\left(\kappa_{u_{\beta}}\right)\right\rangle
$$

to

$$
x^{\prime} \frown s \frown\left\langle w, A_{\kappa_{u_{\beta}}}, H_{\kappa_{u_{\beta}}}, H_{\kappa_{u_{\beta}}}\left(\kappa_{s}\right)\right\rangle \in D,
$$

where $x^{\prime} \in Q_{u_{\beta}} . x^{\prime}$ must be good.

So find a good $x$ in the filter on $Q_{u_{\beta}}$ generated by $(\vec{u}|\beta, \vec{F}| \beta)$. By construction there is a $q \in E_{x}$ which also lies in the filter generated by $\left(\left\langle u_{\gamma}: \beta \leq \gamma<\lambda\right\rangle,\left\langle F_{\gamma}: \beta \leq \gamma<\lambda\right\rangle\right)$. So $x \oplus q \in E$, and this also lies in the filter generated by $\left(\left\langle u_{\gamma}: \gamma<\lambda\right\rangle,\left\langle F_{\gamma}: \gamma<\lambda\right\rangle\right)$. So the latter filter meets every dense set and is therefore generic.

3.6. The forcing preserves successors. We are now able to answer the question which was left unsolved by the Prikry lemma, namely whether the successors of limit points on the generic sequence are collapsed. By the Prikry lemma it is enough to decide whether $Q_{w}$ collapses $\kappa_{w}^{+}$. The proof given here was suggested by Hugh Woodin, and replaces a much clumsier one which used the canonical generic object described in the next section. 
Theorem 1. If $G$ is $Q_{w}$-generic then $\kappa_{w}^{+}$is a cardinal in $V[G]$. Proof. Suppose not, and let $i$ be a term and $p \in Q_{w}$ a condition such that

$$
p \Vdash \dot{\tau}: \kappa_{w} \rightarrow \kappa_{w}^{+} .
$$

Find $\theta$ some large regular cardinal such that $p, \dot{\tau}, Q_{w}$ are in $H_{\theta}$. Find $X \prec H_{\theta}$ such that $V_{\kappa_{w}} \subseteq N$ and $p, \dot{\tau}, Q_{w}$ are in $X$, and such that $|X|=\kappa_{w}$. Let $\pi: X \rightarrow N$ be the Mostowski collapse of $X$ to a transitive set $N$. Force over $H_{\theta}$ with $Q_{w} \mid p$ to get a generic $(\vec{v}, \vec{F})$.

Claim 1. The pair $(\vec{v}, \vec{F})$ is $\pi\left(Q_{w}\right)$-generic over the model $N$.

Proof. This is immediate using the geometric criterion for genericity and the observation that we have

$$
\pi\left(\mathscr{F}_{w}\right)=\mathscr{F}_{w} \cap N, \quad \pi(w(1))=w(1) \cap N .
$$

Let $G$ be the generic filter on $Q_{w}$ corresponding to $(\vec{v}, \vec{F})$, and let $\bar{G}$ be the filter on $\pi\left(Q_{w}\right)$ similarly defined. Observing that $\pi^{-1}$ maps elements of $\bar{G}$ to elements of $G$, we build an elementary embedding

$$
k: N[\bar{G}] \rightarrow H_{\theta}[G] .
$$

But $\pi(p) \in \bar{G}$ so the interpretation of $\dot{\tau}$ by $\bar{G}$ must be $f: \kappa_{w} \rightarrow\left(\kappa_{w}\right)_{N}^{+}$. But $\left(\kappa_{w}\right)_{N}^{+}$is the critical point of $k$ so $k(f)=f$, while by elementarity $k(f): \kappa_{w} \rightarrow \kappa_{w}^{+}$. This gives a contradiction.

3.7. A canonical generic sequence. Radin showed in $[R]$ that generics for Radin forcing can be generated by iterated elementary embeddings; the same is true for $Q_{w}$, with a similar proof (Radin's iterative scheme for choosing the ordinals to which sequences are restricted has the same effect as the one given here).

Fix $w \in U_{\infty}$, with constructing pair $(k, F)$, where $\operatorname{lh}(w)<k\left(\kappa_{w}\right)$. Iterate $k$ to get a sequence of models $\left\langle M_{\alpha}: \alpha \in \mathrm{ON}\right\rangle$ (where $M_{0}=V$ ) and embeddings $k_{\alpha \beta}$ for $\alpha<\beta$ in the usual way. Define $F_{\alpha}=k_{0 \alpha}(F), \kappa_{\alpha}=k_{0 \alpha}\left(\kappa_{w}\right)$ for each $\alpha \in \mathrm{ON}$. It is easily seen that $F_{\alpha}$ is $P\left(\kappa_{\alpha}, \kappa_{\alpha+1}\right)_{M_{\beta}}$-generic over $M_{\beta}$ for $\beta>\alpha$; also if $\alpha<\alpha^{\prime}<\beta$ then $F_{\alpha}$ and $F_{\alpha^{\prime}}$ are mutually generic over $M_{\beta}$. Define by induction a sequence $\left\langle u_{\alpha}\right\rangle$

$$
\begin{aligned}
& u_{\alpha}=\left\langle\kappa_{\alpha}\right\rangle, \quad \alpha \text { zero or a successor, } \\
& u_{\alpha}=k_{0 \alpha}(w)\left\lceil\lambda_{\alpha}, \quad \alpha\right. \text { limit, }
\end{aligned}
$$

where in the limit case $\lambda_{\alpha}$ is the least $\lambda<\operatorname{lh}\left(k_{0 \alpha}(w)\right)$ such that for some $X \in k_{0 \alpha}(w)(\lambda)$ the sequence $\left\langle u_{\beta}: \beta<\alpha\right\rangle$ avoids $X$ if such $\lambda$ exists, and $\lambda_{\alpha}=\operatorname{lh}\left(k_{0 \alpha}(w)\right)$ otherwise. The construction ends at $\alpha$ in the latter case.

Lemma 1. For all $\alpha$ such that $u_{\alpha}$ is defined and $\ln \left(u_{\alpha}\right)>1$ the pair $\left(\left\langle u_{\beta}: \beta<\right.\right.$ $\left.\alpha\rangle,\left\langle F_{\beta}: \beta<\alpha\right\rangle\right)$ is generic over $M_{\alpha}$ for the forcing $Q_{u_{\alpha}}$.

Proof. By induction on $\alpha$. The nontrivial part is to show that the sequence $\left\langle u_{\beta}: \beta<\alpha\right\rangle$ generates the filter $\mathscr{F}_{u_{\alpha}}$. One direction is easy; suppose that $X$ belongs to $M_{\alpha}$ and contains a final segment of $\vec{u}\left\lceil\alpha\right.$, but $X \notin \mathscr{F}_{u_{\alpha}}$. So for some $\gamma<\lambda_{\alpha}, X^{c} \in k_{0 \alpha}(w)(\gamma)$. But by the minimal choice of $\lambda_{\alpha},\left\{\beta<\alpha \mid u_{\beta} \in X^{c}\right\}$ is unbounded in $\alpha$ and this is absurd. 
For the other direction let $X \in \mathscr{F}_{u_{\alpha}}$. Fix $Y \in k_{0 \alpha}(w)\left(\lambda_{\alpha}\right)$ such that $\forall \beta<\alpha$ $u_{\beta} \notin Y$, and define

$$
Z=\left\{v \mid \exists \mu \quad Y \cap V_{\kappa_{v}} \in v(\mu)\right\} .
$$

A familiar style of argument using $k_{\alpha \alpha+1}$ (which generates the sequence $k_{0 \alpha}(w)$ ) shows

$$
T=X \cup Y \cup Z \in \mathscr{F}_{k_{0 \alpha}(w)} ;
$$

$\alpha$ is limit, so by the definition of direct limit for all large $\beta<\alpha \quad T=k_{\beta \alpha}\left(T_{\beta}\right)$, where $T_{\beta} \in \mathscr{T}_{k_{0 \beta}(w)}$. But $k_{\beta \beta+1}$ generates $k_{0 \beta}(w)$, therefore for such $\beta$

$$
\forall \gamma<\operatorname{lh}\left(k_{0 \beta}(w)\right) k_{0 \beta}(w)\left\lceil\gamma \in T_{\beta+1} .\right.
$$

$k_{\beta+1 \alpha}$ fixes the sequence $k_{0 \beta}(w) \backslash \gamma$, because

$$
\operatorname{lh}\left(k_{0 \beta}(w)\right)<k_{0 \beta+1}\left(\kappa_{0}\right)=\operatorname{crit}\left(k_{0 \beta+1}\right) .
$$

So

$$
\forall \gamma<\ln \left(k_{0 \beta}(w)\right) k_{0 \beta}(w)\lceil\gamma \in T .
$$

As the construction did not halt at $\beta, u_{\beta}$ is a proper initial segment of $k_{0 \beta}(w)$, so $u_{\beta} \in T$. But now it must be the case that $u_{\beta} \in X$; for certainly $u_{\beta} \notin Y$, while if $u_{\beta} \in Z$ then (as by induction $\left\langle u_{\gamma}: \gamma<\beta\right\rangle$ is $Q_{u_{\beta}}$-generic over $M_{\beta}$ ) there is $\gamma<\beta, u_{\gamma} \in Y$.

Theorem 1. The construction of the $u_{\alpha}$ terminates at some stage.

Proof. Suppose not. Fix $\mu$ a large enough regular cardinal and select $X_{\alpha} \in$ $k_{0 \alpha}(w)\left(\lambda_{\alpha}\right)$ for each limit $\alpha \leq \mu$ such that $\vec{u} \mid \alpha$ avoids $X_{\alpha}$. A familiar argument from the theory of inner models (see, e.g., Lemma 4 of Mitchell's paper [Mi1]) shows that for some stationary set $S \subset \mu$

$$
\forall(\alpha, \beta) \in[S]^{2} \quad k_{0 \alpha}\left(\lambda_{\alpha}\right)=\lambda_{\beta} \wedge k_{0 \alpha}\left(X_{\alpha}\right)=X_{\beta}
$$

Fix such a pair $(\alpha, \beta) . X_{\alpha} \in k_{0 \alpha}(w)\left(\lambda_{\alpha}\right)$, so arguing as above

$$
u_{\alpha}=k_{0 \alpha}(w)\left\lceil\lambda_{\alpha} \in k_{\alpha \beta}\left(X_{\alpha}\right)=X_{\beta},\right.
$$

contradicting the choice of $X_{\beta}$.

\subsection{Repeat points and the preservation of large cardinals.}

Definition. Let $w \in U_{\infty} . \alpha<\operatorname{lh}(w)$ is a repeat point for $w$ iff

$$
\forall X \quad X \in w(\alpha) \Rightarrow \exists \beta<\alpha \quad X \in w(\beta) .
$$

Lemma 1. If $\ln (w) \geq\left(2^{\kappa_{w}}\right)^{+}$then $w$ has a repeat point.

Proof. For each $\nu<\operatorname{lh}(w)$ which is not a repeat, fix (by the definition of repeat point) $X_{\nu} \in w(\nu) \backslash \bigcup_{\mu<\nu} w(\mu)$. There are only $2^{\kappa_{w}}$ possible $X_{\nu}$.

Lemma 2. If $\beta$ is a repeat point for $w$ and $A \in \mathscr{F}_{w\lceil\beta}$ then $A \in w(\beta)$.

Proof. Suppose not: then $A^{c} \in w(\beta)$, so as $\beta$ is a repeat point there is $\alpha<\beta$ with $A^{c} \in w(\alpha)$. But this is absurd.

Lemma 3. Let $w \in U_{\infty}$, with $(i: V \rightarrow M, g)$ a constructing pair and $\operatorname{lh}(w)<$ $i\left(\kappa_{w}\right)$. Let $\beta<\ln (w)$ be a repeat for $w$, and $v=w\lceil\beta$. Let

$$
p=x \frown\langle v, A, H, h\rangle \in Q_{v} .
$$


Then

$$
p \frown\left\langle i(v), i(A), i(H), i(H)\left(\kappa_{v}\right)\right\rangle \leq_{i\left(Q_{v}\right)} i(p) .
$$

Proof. It is required to show that $\langle v, A, H, h\rangle$ can be added to $i(p)=x \frown$ $\langle i(v), i(A), i(H), i(h)\rangle . \quad A \in \mathscr{F}_{v}$, so by Lemma $2 A \in w(\beta)$ and therefore $v=w \mid \beta \in i(A)$. Also $A=i(A) \cap V_{\kappa_{v}}, H=i(H) \uparrow\left[\kappa_{v}\right]^{2}$, and $h=i(h) \uparrow \kappa_{v}$.

The lemma is proved.

Now we use the canonical generic sequence constructed in the last section to show that the forcing $Q_{w}$ can preserve large cardinal properties of $\kappa_{w}$. It is possible to do this proof in the style of [R], using the Prikry lemma to define extenders in the generic extension, but the proof we give here is rather shorter and more perspicuous.

Theorem 1. With the same assumptions as in Lemma 3 let $G$ be generic for $Q_{w\lceil\beta}$, and do the construction from the last subsection to produce an $\alpha$ and an $H$ such that $H$ is $i_{0 \alpha}\left(Q_{w\lceil\beta}\right)$-generic over $M_{\alpha}$, where $i_{0 \alpha}: V \rightarrow M_{\alpha}$ is the $\alpha$ th iterate of $i$. Write $G=(\vec{x}, \vec{E})$, where $\vec{x}$ is a sequence of measure sequences and $\vec{E}$ is an appropriate sequence of generic collapses; similarly let $H=(\vec{u}, \vec{F})$, and define a new pair $G+H=(\vec{v}, \vec{I})$, where

$$
\vec{v}=\vec{x} \frown\left\langle w\lceil\beta\rangle \frown \vec{u} \uparrow\left(\operatorname{dom}(\vec{u}) \backslash\left\{\kappa_{w}\right\}\right), \quad \vec{I}=\vec{E} \frown \vec{F} .\right.
$$

Then $G+H$ is $i_{0 \alpha}\left(Q_{w\lceil\beta}\right)$-generic over $M_{\alpha}$, and

$$
\forall p \quad p \in G \Leftrightarrow i_{0 \alpha}(p) \in G+H .
$$

Proof. The genericity is immediate by the geometric condition, so fix $p=x \frown$ $\langle w\lceil\beta, A, H, h\rangle$ with $p \in G$. By the geometric condition it suffices to show that $p \frown\left\langle i_{0 \alpha}(v), i_{0 \alpha}(A), i_{0 \alpha}(H), i_{0 \alpha}(H)\left(\kappa_{w}\right)\right\rangle \in G+H$, which amounts to showing that the sequences (excepting the first) from $\vec{u}$ and the generics from $\vec{F}$ are controlled by the top part of this condition. By construction each sequence occurring on $\vec{u}$ is a proper subsequence of some $i_{0 \gamma}(w\lceil\beta)$ for some $\gamma$, say $i_{0 \gamma}(w) \uparrow \lambda$. As $A$ is in all the measures on $w\left\lceil\beta, i_{0 \gamma}(A) \in i_{0 \gamma}(w)(\lambda)\right.$ by elementarity, hence $i_{0 \gamma}(w) \mid \lambda \in i_{0 \gamma+1}(A)$; then $i_{0 \gamma}(w) \backslash \lambda \in i_{0 \alpha}(A)$ because $i_{\gamma+1 \alpha}$ has large critical point. That the entries of $\vec{F}$ are controlled by the top part is immediate from the observation that $i_{02}(H)\left(\kappa_{w}, i\left(\kappa_{w}\right)\right) \in g$.

Now it is possible to extend the map $i_{0 \alpha}: V \rightarrow M_{\alpha}$ to a map $i^{*}: V[G] \rightarrow$ $M_{\alpha}[G+H]$, so that certainly the measurability of $\kappa_{w}$ is preserved. In fact more is true, for observe that if $\left(\kappa_{w}\right)^{+}<\lambda<i\left(\kappa_{w}\right)$ and $\mathscr{P} \lambda \subset M$ then $\mathscr{P} \lambda \subset M_{\alpha}$, because $\operatorname{crit}\left(i_{1 \alpha}\right)=i\left(\kappa_{w}\right)>\lambda$. An easy chain condition argument shows that if this is the case then

$$
(\mathscr{P} \lambda)_{V[G]} \subset M_{\alpha}[G+H]
$$

so that we can preserve any strength that the embedding $i$ may happen to have.

\subsection{The final model.}

Main Theorem. If it is consistent that GCH holds and there is a $\mathscr{P}_{3} \kappa$-hypermeasurable cardinal, then it is consistent that GCH hold at every successor and fail at every limit. 
Proof. The proof of the Main Theorem is now straightforward. We perform the Reverse Easton construction of $\S 2$ to get the initial hypotheses of this section. Then using the constructing pair $(j, F)$ we build a sequence $u$ with one repeat point $\beta$. We force with $Q_{u \uparrow \beta}$, and then truncate the model $V[G]$ (in which $\kappa$ is $\mathscr{P}_{2} \kappa$-hypermeasurable) at $\kappa$. The result is a set model of ZFC in which GCH holds at every successor and fails at every limit, containing many moderately large cardinals.

\section{REFERENCES}

[B] J. Baumgartner, Iterated forcing, Surveys in Set Theory, London Math. Soc. Lecture Notes, vol. 87, Cambridge Univ. Press, Cambridge, 1983, pp. 1-55.

[Ca] G. Cantor, Ein Beitrag zur Mannigfaltigskeitslehre, J. Reine Angew. Math. 84 (1878), 242-258.

[C] J. Cummings, Consistency results on cardinal exponentiation, Cambridge University, 1988.

[D] A. Dodd, The core model, London Math. Soc. Lecture Notes, vol. 61, Cambridge Univ. Press, Cambridge, 1982.

[DJ] K. Devlin and R. Jensen, Marginalia to a theorem of Silver, Logic conference Kiel 1974, Lecture Notes in Math. vol. 499, Springer, Berlin, 1974, pp. 115-142.

[E] W. Easton, Powers of regular cardinals, Ann. Math. Logic 1 (1964), 139-178.

[FW] M. Foreman and W. H. Woodin (to appear).

[GM] M. Gitik and M. Magidor (to appear).

[J] T. Jech, Set theory, Pure and Appl. Math., vol. 79, Academic Press, New York, 1978.

[K] K. Kunen, Set theory, North-Holland, Amsterdam, 1980.

[M] A. Mathias, Sequences generic in the sense of Prikry, J. Austral. Math. Soc. 15 (1973), 409-414.

[Ma1] M. Magidor, On the singular cardinals problem. I, Israel J. Math. 28 (1977), 1-31.

[Ma2] - On the singular cardinals problem. II, Ann. of Math. (2) 106 (1977), 517-549.

[Mil] W. Mitchell, Sets constructed from sequences of measures: revisited, J. Symbolic Logic 48 (1983), 600-609.

[Mi2] _ The core model for sequences of measures. I, Math. Proc. Cambridge Philos. Soc. 95 (1984), 229-260.

[P] K. Prikry, Changing measurable into accessible cardinals, Dissertationes Math. 68 (1970), $5-52$.

[R] L. Radin, Adding closed cofinal sequences to large cardinals, Ann. Math. Logic 22 (1982), 243-261.

[Sc] D. Scott, Measurable cardinals and constructible sets, Bull. Acad. Polon. Sci. Sér. Sci. Math. 9 (1961), 521-524.

[Sh1] S. Shelah, Proper forcing, Lecture Notes in Math., vol. 940, Springer, Berlin, 1982.

[Sh2] _ The singular cardinals problem: independence results, Surveys in Set Theory, London Math. Soc. Lecture Notes, vol. 87, Cambridge Univ. Press, Cambridge, 1983, pp. 116-134.

[Si1] J. Silver, On the singular cardinals problem, Proc. Internat. Congr. Math., Vancouver, 1974, pp. 115-142.

[Si2] _ Unpublished notes on reverse Easton forcing.

[W] W. H. Woodin (to appear).

Mathematical Sciences Research Institute, 1000 Centennial Drive, Berkeley, CaliFORNIA 94720

Current address: Department of Mathematics, Massachusetts Institute of Technology, Cambridge, Massachusetts 02139 